\title{
Next-to-next-to-next-to-leading-order soft-gluon corrections in hard-scattering processes near threshold
}

\author{
Nikolaos Kidonakis \\ Kennesaw State University, Physics \#1202 \\ 1000 Chastain Rd., Kennesaw, GA 30144-5591
}

\begin{abstract}
I present a unified calculation of soft-gluon corrections to hard-scattering cross sections through next-to-next-to-next-to-leading order (NNNLO). Master formulas are derived, from a threshold resummation formalism, that can be applied to total and differential cross sections for hard-scattering processes in hadron colliders. I also present numerical results for charged Higgs production at the LHC where these corrections are large, and for top quark production at the Tevatron where these corrections greatly reduce the scale dependence of the cross section.
\end{abstract}




\section{Introduction}

Calculations of hard-scattering cross sections become very complicated as one moves from the lowest order to higher-order corrections. Current theoretical approaches include a variety of resummations and fixed-order calculations, some through next-to-next-to-leading order (NNLO) [1. These tools can greatly improve next-to-leading order (NLO) calculations because higherorder corrections reduce the scale dependence and increase theoretical accuracy.

The QCD corrections can be separated into hard, soft, and virtual parts, corresponding to contributions from energetic, soft, and virtual gluons, respectively. The soft-gluon corrections are an important component of the total result and, in some schemes and kinematical regions, e.g. threshold, they are numerically dominant. There is a universality in the form of these softgluon corrections, as can be clearly seen from the techniques of threshold resummations, which formally resum the soft-gluon contributions to all orders in the strong coupling [2, 3, 4, 5, 6, 7, 8, . Although resummed calculations are prescription-dependent (see discussion in Ref. [9]), finiteorder expansions of resummed cross sections are not, and they have provided us with many cross sections with NNLO soft-gluon corrections (for a review see Ref. [10]). Examples include $W$ boson production at large transverse momentum [11, direct photon production [12, top quark production [9, 13, 14, bottom and charm quark production [15], heavy quark electroproduction [16] and photoproduction [17, charged Higgs production [18, and jet production [19].

The NLO soft-gluon corrections are typically a very good approximation to the exact NLO corrections near threshold. The NNLO soft-gluon corrections can be numerically significant and they invariably improve the theoretical calculation by stabilizing the dependence of the cross section on the factorization and renormalization scales, which are arbitrary energy scales in the theory. Thus it is worthwhile to provide a unified approach for the calculation of these and even higher-order soft-gluon corrections to hard-scattering processes in hadron colliders. It is important to note that new particles, such as in supersymmetry [20] or Higgs physics [21], or particle production via new interactions, such as top production via flavor-changing neutral currents [22, will likely be discovered near threshold where the soft-gluon corrections are dominant, therefore soft-gluon calculations are relevant to more than just pure QCD processes.

The calculation of hard-scattering cross sections in hadron-hadron or lepton-hadron collisions can be written as

$$
\sigma=\sum_{f} \int\left[\prod_{i} d x_{i} \phi_{f / h_{i}}\left(x_{i}, \mu_{F}\right)\right] \hat{\sigma}\left(s, t_{i}, \mu_{F}, \mu_{R}\right),
$$

where $\sigma$ is the physical cross section, $\phi_{f / h_{i}}$ is the distribution function for parton $f$ carrying momentum fraction $x_{i}$ of hadron $h_{i}$, at a factorization scale $\mu_{F}$, and $\mu_{R}$ is the renormalization scale. The parton-level cross section is denoted by $\hat{\sigma}$, and $s, t_{i}$ are standard kinematical invariants formed from the 4-momenta of the particles in the hard scattering. In a lepton-hadron collision we have one parton distribution $(i=1)$ while in a hadron-hadron collision we have two, $i=1,2$. The partonic processes are of the form

$$
f_{1}\left(p_{1}\right)+f_{2}\left[l_{2}\right]\left(p_{2}\right) \rightarrow F+X
$$

where $f[l]$ represents a parton [lepton], $F$ represents an observed system in the final state, and $X$ any additional allowed final-state particles. For example, $F$ can represent a pair of 
heavy quarks, a single heavy quark, a jet, a photon, a Higgs boson, a pair of squarks, etc. Then $s=\left(p_{1}+p_{2}\right)^{2}$. In single-particle-inclusive (1PI) kinematics we identify one particle $F$ with momentum $p$, and define the kinematical invariants $t_{1}=\left(p_{1}-p\right)^{2}, t_{2}=\left(p_{2}-p\right)^{2}$ (also commonly denoted by $t$ and $u$, respectively). In pair-invariant-mass (PIM) kinematics we identify a pair of particles (such as a heavy quark antiquark pair) with invariant mass squared $Q^{2}$. We note here that $\sigma$ and $\hat{\sigma}$ are not restricted to be total cross sections; they can represent any differential cross section of interest.

In general, $\hat{\sigma}$ includes plus distributions $\mathcal{D}_{l}\left(x_{t h}\right)$ with respect to a kinematical variable $x_{t h}$ that measures distance from threshold, with $l \leq 2 n-1$ at $n$th order in $\alpha_{s}$ beyond the leading order. These are the soft corrections. The virtual corrections multiply delta functions $\delta\left(x_{t h}\right)$. In 1PI kinematics, $x_{t h}$ is usually denoted as $s_{4}$ (or $s_{2}$ ), $s_{4}=s+t_{1}+t_{2}-\sum m^{2}$ (the sum is over the masses squared of all particles in the process), and it vanishes at threshold. The plus distributions are of the form

$$
\mathcal{D}_{l}\left(s_{4}\right) \equiv\left[\frac{\ln ^{l}\left(s_{4} / M^{2}\right)}{s_{4}}\right]_{+},
$$

where $M^{2}$ is a hard scale relevant to the process at hand, for example the mass $m$ of a heavy quark, the transverse momentum $p_{T}$ of a jet, etc. The distributions are defined through their integral with any smooth function, such as parton densities, by

$$
\begin{aligned}
\int_{0}^{s_{4 \max }} d s_{4} f\left(s_{4}\right)\left[\frac{\ln ^{l}\left(s_{4} / M^{2}\right)}{s_{4}}\right]_{+} \equiv & \int_{0}^{s_{4 \max }} d s_{4} \frac{\ln ^{l}\left(s_{4} / M^{2}\right)}{s_{4}}\left[f\left(s_{4}\right)-f(0)\right] \\
& +\frac{1}{l+1} \ln ^{l+1}\left(\frac{s_{4 \max }}{M^{2}}\right) f(0) .
\end{aligned}
$$

In PIM kinematics, with $Q^{2}$ the invariant mass squared of the produced pair, $x_{t h}$ is usually called $1-x$ or $1-z$, with $z=Q^{2} / s \rightarrow 1$ at threshold. Then the plus distributions are of the form

$$
\mathcal{D}_{l}(z) \equiv\left[\frac{\ln ^{l}(1-z)}{1-z}\right]_{+}
$$

defined through their integral with any smooth function by

$$
\begin{aligned}
\int_{y}^{1} d z f(z)\left[\frac{\ln ^{l}(1-z)}{1-z}\right]_{+} \equiv & \int_{y}^{1} d z \frac{\ln ^{l}(1-z)}{1-z}[f(z)-f(1)] \\
& +\frac{1}{l+1} \ln ^{l+1}(y) f(1)
\end{aligned}
$$

The highest powers of these distributions in the $n$ th-order corrections are the leading logarithms (LL) with $l=2 n-1$, the second highest are the next-to-leading logarithms (NLL) with $l=$ $2 n-2$, the third highest are the next-to-next-to-leading logarithms (NNLL) with $l=2 n-3$, the fourth highest are the next-to-next-to-next-to-leading logarithms (NNNLL) with $l=2 n-4$, etc. These logarithms can be in principle resummed to all orders in perturbation theory. By now there are several processes for which NLL resummations and NNLO-NNLL results (i.e. the NNLL terms ${ }^{1}$ at NNLO) have been presented [10, 23.

\footnotetext{
${ }^{1}$ The counting of logarithms is different in the exponent and in the fixed-order expansions; for example a term that is NNLL in the fixed-order expansion, as described here, may be NLL in the exponent [13].
} 
In this paper I present master formulas for the NLO, NNLO, and NNNLO soft-gluon corrections for processes in hadron-hadron or hadron-lepton collisions. These processes can be of QCD, electroweak, Higgs, or supersymmetric origin at lowest order. Results on the NLO and NNLO corrections have been presented before [23] but the notation here is somewhat different to facilitate the calculation of the NNNLO corrections. The NNNLO results and their applications to top quark and charged Higgs production are new. In the next section, I present a threshold resummation formula from which high-order expansions are derived. In Sections 3 and 4 are presented master formulas for the NLO and NNLO soft corrections, respectively, that arise from the expansion of the resummation formula. The formulas are given in the $\overline{\mathrm{MS}}$ scheme (see 23] for results through NNLO in the DIS scheme), and cover both 1PI and PIM kinematics. In Section 5, I present a master formula for the NNNLO corrections. In Sections 6 and 7 applications to charged Higgs production at the LHC and top quark pair production at the Tevatron, respectively, are discussed. Conclusions are given in Section 8. Some long expressions for terms in the NNNLO master formula are collected in an Appendix.

\section{Soft-gluon corrections from threshold resummation}

We begin with a brief review of the threshold resummation formalism. Threshold resummation follows from factorization theorems for hard-scattering cross sections. One can write the hadronic cross section as a convolution of parton densities with a parton-level cross section. This can be further refactorized into functions associated with soft and collinear gluon emission from the incoming partons and any outgoing partons or jets, a function associated with noncollinear soft gluon emission that involves the color structure of the hard scattering, and a short-distance hard-scattering function. The renormalization group properties of these functions result in the exponentiation of the soft-gluon contributions thus providing the resummed cross section [5, 6, 7]. For a review see Ref. [24].

The resummation of threshold logarithms is carried out in moment space. We define moments of the partonic cross section by $\hat{\sigma}(N)=\int d z z^{N-1} \hat{\sigma}(z)(\mathrm{PIM})$ or by $\hat{\sigma}(N)=\int\left(d s_{4} / s\right) e^{-N s_{4} / s} \hat{\sigma}\left(s_{4}\right)$ (1PI), with $N$ the moment variable. The logarithms of $N$ exponentiate. The resummed partonic cross section in moment space is then given by

$$
\begin{aligned}
& \hat{\sigma}^{r e s}(N)=\exp \left[\sum_{i} E^{f_{i}}\left(N_{i}\right)\right] \exp \left[\sum_{j} E^{\prime f_{j}}\left(N_{j}\right)\right] \\
& \times \exp \left[\sum_{i} 2 \int_{\mu_{F}}^{\sqrt{s}} \frac{d \mu}{\mu} \gamma_{i / i}\left(\alpha_{s}(\mu)\right)\right] \exp \left[2 d_{\alpha_{s}} \int_{\mu_{R}}^{\sqrt{s}} \frac{d \mu}{\mu} \beta\left(\alpha_{s}(\mu)\right)\right] \\
& \times \operatorname{Tr}\left\{H^{f_{i} f_{j}}\left(\alpha_{s}\left(\mu_{R}\right)\right) \exp \left[\int_{\sqrt{s}}^{\sqrt{s} / \tilde{N}_{j}} \frac{d \mu}{\mu} \Gamma_{S}^{\dagger f_{i} f_{j}}\left(\alpha_{s}(\mu)\right)\right] \tilde{S}^{f_{i} f_{j}}\left(\alpha_{s}\left(\sqrt{s} / \tilde{N}_{j}\right)\right)\right. \\
&\left.\times \exp \left[\int_{\sqrt{s}}^{\sqrt{s} / \tilde{N}_{j}} \frac{d \mu}{\mu} \Gamma_{S}^{f_{i} f_{j}}\left(\alpha_{s}(\mu)\right)\right]\right\} .
\end{aligned}
$$

The sums over $i$ run over incoming partons: in hadron-hadron colisions we have two partons in the initial state, so $i=1,2$; in lepton-hadron collisions we have one parton. The sum over $j$ 
is relevant if we have massless partons in the final state at lowest order. We note that we have suppressed all gauge-dependent terms because these terms cancel out explicitly.

Equation (2.1) is actually valid for both 1PI and PIM kinematics with appropriate definitions for $N_{i}$ and $N_{j}$. In 1PI kinematics $N_{i}=N\left(-t_{i} / M^{2}\right)$ for incoming partons $i$, and $N_{j}=N\left(s / M^{2}\right)$ for outgoing partons $j$; here $M^{2}$ is any chosen hard scale relevant to the process at hand. In PIM kinematics $N_{i}=N_{j}=N$. Also note that $\tilde{N}=N e^{\gamma_{E}}$, with $\gamma_{E}$ the Euler constant. The various exponents above are known at most to three loops. Below we give explicitly only the one-loop (and some two-loop) expressions that will be needed in our applications to charged Higgs production at NLL accuracy and top quark production at NNLL accuracy. Some two-loop and three-loop results can be found explicitly in [23] and [25, 26]. We note however that the two-loop and higher-loop results for process-dependent functions, such as the soft anomalous dimensions $\Gamma_{S}$, have to be calculated explicitly for a specified partonic process.

The first exponent in Eq. (2.1) is given in the $\overline{\mathrm{MS}}$ scheme by

$$
E^{f_{i}}\left(N_{i}\right)=-\int_{0}^{1} d z \frac{z^{N_{i}-1}-1}{1-z}\left\{\int_{(1-z)^{2}}^{1} \frac{d \lambda}{\lambda} A_{i}\left(\alpha_{s}(\lambda s)\right)+\nu_{i}\left[\alpha_{s}\left((1-z)^{2} s\right)\right]\right\}
$$

with $A_{i}\left(\alpha_{s}\right)=A_{i}^{(1)} \alpha_{s} / \pi+A_{i}^{(2)}\left(\alpha_{s} / \pi\right)^{2}+A_{i}^{(3)}\left(\alpha_{s} / \pi\right)^{3}+\cdots$. Here $A_{i}^{(1)}=C_{i}$ with $C_{i}=C_{F}=$ $\left(N_{c}^{2}-1\right) /\left(2 N_{c}\right)$ for a quark or antiquark and $C_{i}=C_{A}=N_{c}$ for a gluon, with $N_{c}$ the number of colors, while $A_{i}^{(2)}=C_{i} K / 2$ with $K=C_{A}\left(67 / 18-\pi^{2} / 6\right)-5 n_{f} / 9$ [27], where $n_{f}$ is the number of quark flavors. Also $\nu_{i}=\left(\alpha_{s} / \pi\right) \nu_{i}^{(1)}+\left(\alpha_{s} / \pi\right)^{2} \nu_{i}^{(2)}+\left(\alpha_{s} / \pi\right)^{3} \nu_{i}^{(3)}+\cdots$, with $\nu_{i}^{(1)}=C_{i}$.

The second exponent is given by

$$
E^{\prime f_{j}}\left(N_{j}\right)=\int_{0}^{1} d z \frac{z^{N_{j}-1}-1}{1-z}\left\{\int_{(1-z)^{2}}^{1-z} \frac{d \lambda}{\lambda} A_{j}\left(\alpha_{s}(\lambda s)\right)-B_{j}\left[\alpha_{s}((1-z) s)\right]-\nu_{j}\left[\alpha_{s}\left((1-z)^{2} s\right)\right]\right\},
$$

where $B_{j}=\left(\alpha_{s} / \pi\right) B_{j}^{(1)}+\left(\alpha_{s} / \pi\right)^{2} B_{j}^{(2)}+\left(\alpha_{s} / \pi\right)^{3} B_{j}^{(3)}+\cdots$ with $B_{q}^{(1)}=3 C_{F} / 4$ and $B_{g}^{(1)}=\beta_{0} / 4$, where $\beta_{0}$ is the lowest-order $\beta$-function, $\beta_{0}=\left(11 C_{A}-2 n_{f}\right) / 3$.

In the third exponent $\gamma_{i / i}$ is the moment-space anomalous dimension of the $\overline{\mathrm{MS}}$ density $\phi_{i / i}$. Note that the $N$-independent part of the one-loop $\gamma_{i / i}$ is the same as $\gamma_{i}^{(1)}$, the one-loop parton anomalous dimension, given by $\gamma_{q}^{(1)}=3 C_{F} / 4$ and $\gamma_{g}^{(1)}=\beta_{0} / 4$ for quarks and gluons, respectively 13 .

The $\beta$ function in the fourth exponent is given in the Appendix. The constant $d_{\alpha_{s}}=0,1,2$ if the Born cross section is of order $\alpha_{s}^{0}, \alpha_{s}^{1}, \alpha_{s}^{2}$, respectively.

$H^{f_{i} f_{j}}$ are the hard-scattering functions for the scattering of partons $f_{i}$ and $f_{j}$, while $S^{f_{i} f_{j}}$ are the soft functions describing noncollinear soft gluon emission. We use the expansions $H=\alpha_{s}^{d_{\alpha_{s}}} H^{(0)}+\left(\alpha_{s}^{d_{\alpha_{s}}+1} / \pi\right) H^{(1)}+\left(\alpha_{s}^{d_{\alpha_{s}}+2} / \pi^{2}\right) H^{(2)}+\left(\alpha_{s}^{d_{\alpha_{s}}+3} / \pi^{3}\right) H^{(3)}+\cdots$ and $S=S^{(0)}+$ $\left(\alpha_{s} / \pi\right) S^{(1)}+\left(\alpha_{s} / \pi\right)^{2} S^{(2)}+\left(\alpha_{s} / \pi\right)^{3} S^{(3)}+\cdots$. Note that both $H$ and $S$ are matrices in color space and the trace is taken. At lowest order, the trace of the product of the hard matrices $H$ and soft matrices $S$ reproduces the Born cross section for each partonic process, $\sigma^{B}=\alpha_{s}^{d_{\alpha_{s}}} \operatorname{tr}\left[H^{(0)} S^{(0)}\right]$. The evolution of the soft function follows from its renormalization group properties and is given in terms of the soft anomalous dimension matrix $\Gamma_{S}$ [5, 6, 24]. In processes with simple color flow $\Gamma_{S}$ is a trivial $1 \times 1$ matrix while in processes with complex color flow an appropriate choice of color basis has to be made. For quark-(anti)quark scattering, $\Gamma_{S}$ is a $2 \times 2$ matrix [5, 28]; for 
quark-gluon scattering it is a $3 \times 3$ matrix [6] for gluon-gluon scattering it is an $8 \times 8$ matrix [6]. For the discussion below we expand $\Gamma_{S}$ as $\Gamma_{S}=\left(\alpha_{s} / \pi\right) \Gamma_{S}^{(1)}+\left(\alpha_{s} / \pi\right)^{2} \Gamma_{S}^{(2)}+\left(\alpha_{s} / \pi\right)^{3} \Gamma_{S}^{(3)}+\cdots$. The process-dependent soft anomalous dimension matrices have by now been presented at one loop for all $2 \rightarrow 2$ partonic processes; a compilation of results is given in [24]. They can be explicitly calculated for any process through the calculation of eikonal vertex corrections using the techniques and results in Refs. [5, 6, 24]. Some work has been done on two-loop calculations of these anomalous dimensions [29], and furthermore the universal components of these anomalous dimensions for quark-antiquark and gluon-gluon initiated processes have been extracted from the NNLO results for Drell-Yan and Higgs production as detailed in Ref. [23].

The exponentials in the resummed cross section can be expanded to any fixed order in $\alpha_{s}$ and then inverted to momentum space to provide explicit results for the higher-order corrections. A fixed-order expansion avoids the problems with infrared singularities in the exponents and thus no prescription is needed to deal with these in our approach (see discussion in Ref. [9]).

\section{$3 \quad$ NLO master formula for soft-gluon corrections}

We first expand the resummed formula in Eq. (2.1) to next-to-leading order and present a master formula for the NLO soft-gluon corrections in the $\overline{\mathrm{MS}}$ scheme and 1PI kinematics:

$$
\hat{\sigma}^{(1)}=\sigma^{B} \frac{\alpha_{s}\left(\mu_{R}^{2}\right)}{\pi}\left\{c_{3} \mathcal{D}_{1}\left(s_{4}\right)+c_{2} \mathcal{D}_{0}\left(s_{4}\right)+c_{1} \delta\left(s_{4}\right)\right\}+\frac{\alpha_{s}^{d_{\alpha_{s}}+1}\left(\mu_{R}^{2}\right)}{\pi}\left[A^{c} \mathcal{D}_{0}\left(s_{4}\right)+T_{1}^{c} \delta\left(s_{4}\right)\right],
$$

where $\sigma^{B}$ is the Born term,

$$
c_{3}=\sum_{i} 2 C_{i}-\sum_{j} C_{j}
$$

with $C_{q}=C_{F}$ and $C_{g}=C_{A}$, and $c_{2}$ is defined by $c_{2}=c_{2}^{\mu}+T_{2}$, with

$$
c_{2}^{\mu}=-\sum_{i} C_{i} \ln \left(\frac{\mu_{F}^{2}}{M^{2}}\right)
$$

denoting the terms involving logarithms of the scale, and

$$
T_{2}=-\sum_{i}\left[C_{i}+2 C_{i} \ln \left(\frac{-t_{i}}{M^{2}}\right)+C_{i} \ln \left(\frac{M^{2}}{s}\right)\right]-\sum_{j}\left[B_{j}^{(1)}+C_{j}+C_{j} \ln \left(\frac{M^{2}}{s}\right)\right]
$$

denoting the scale-independent terms. We remind the reader that the sums over $i$ run over incoming partons and the sums over $j$ run over any massless partons in the final state. Note that not all the NLO corrections are proportional to the Born term; only the leading logarithms and terms involving the scale are. The function $A^{c}$ is process-dependent and depends on the color structure of the hard-scattering. It is defined by

$$
A^{c}=\operatorname{tr}\left(H^{(0)} \Gamma_{S}^{(1) \dagger} S^{(0)}+H^{(0)} S^{(0)} \Gamma_{S}^{(1)}\right) .
$$

With regard to the $\delta\left(x_{t h}\right)$ terms, we split them into a term $c_{1}$, that is proportional to the Born cross section, and a term $T_{1}^{c}$ that is not. $c_{1}=c_{1}^{\mu}+T_{1}$, with

$$
c_{1}^{\mu}=\sum_{i}\left[C_{i} \ln \left(\frac{-t_{i}}{M^{2}}\right)-\gamma_{i}^{(1)}\right] \ln \left(\frac{\mu_{F}^{2}}{M^{2}}\right)+d_{\alpha_{s}} \frac{\beta_{0}}{4} \ln \left(\frac{\mu_{R}^{2}}{M^{2}}\right)
$$


denoting the terms involving logarithms of the scale. $T_{1}$ and $T_{1}^{c}$ do not involve the factorization and renormalization scales. Note that $T_{1}$ and $T_{1}^{c}$ are virtual terms and cannot be derived from the resummation formalism, but they can be read off by matching to a full NLO calculation for any specified process.

In PIM kinematics we simply replace $s_{4}$ by $1-z$, set $s=M^{2}$, and delete all $\ln \left(-t_{i} / M^{2}\right)$ terms from the above expressions. The same should be done for the NNLO and NNNLO results that follow.

As shown in Ref. [23] the NLO master formula passes a number of tests. Its predictions agree with NLO soft-gluon results for all processes where those results are already available.

Also, the renormalization and factorization scale dependence in the physical cross section (after convoluting the partonic cross section with the parton distributions) cancels out explicitly, i.e. $d \sigma / d \mu_{F}=0$ and $d \sigma / d \mu_{R}=0$ at NLO.

\section{NNLO master formula for soft-gluon corrections}

At next-to-next-to-leading order, the expansion of Eq. (2.1), with matching to the NLO softplus-virtual result, Eq. (3.1), gives the NNLO soft corrections in the $\overline{\mathrm{MS}}$ scheme and $1 \mathrm{PI}$ kinematics:

$$
\begin{aligned}
& \hat{\sigma}^{(2)}=\sigma^{B} \frac{\alpha_{s}^{2}\left(\mu_{R}^{2}\right)}{\pi^{2}} \frac{1}{2} c_{3}^{2} \mathcal{D}_{3}\left(s_{4}\right) \\
& +\sigma^{B} \frac{\alpha_{s}^{2}\left(\mu_{R}^{2}\right)}{\pi^{2}}\left\{\frac{3}{2} c_{3} c_{2}-\frac{\beta_{0}}{4} c_{3}+\sum_{j} C_{j} \frac{\beta_{0}}{8}\right\} \mathcal{D}_{2}\left(s_{4}\right)+\frac{\alpha_{s}^{d_{\alpha_{s}}+2}\left(\mu_{R}^{2}\right)}{\pi^{2}} \frac{3}{2} c_{3} A^{c} \mathcal{D}_{2}\left(s_{4}\right) \\
& +\sigma^{B} \frac{\alpha_{s}^{2}\left(\mu_{R}^{2}\right)}{\pi^{2}} C_{D_{1}}^{(2)} \mathcal{D}_{1}\left(s_{4}\right)+\frac{\alpha_{s}^{d_{\alpha_{s}}+2}\left(\mu_{R}^{2}\right)}{\pi^{2}}\left\{\left(2 c_{2}-\frac{\beta_{0}}{2}\right) A^{c}+c_{3} T_{1}^{c}+F^{c}\right\} \mathcal{D}_{1}\left(s_{4}\right) \\
& +\sigma^{B} \frac{\alpha_{s}^{2}\left(\mu_{R}^{2}\right)}{\pi^{2}} C_{D_{0}}^{(2)} \mathcal{D}_{0}\left(s_{4}\right) \\
& +\frac{\alpha_{s}^{d_{\alpha_{s}}+2}\left(\mu_{R}^{2}\right)}{\pi^{2}}\left\{\left[c_{1}-\zeta_{2} c_{3}+\frac{\beta_{0}}{4} \ln \left(\frac{\mu_{R}^{2}}{M^{2}}\right)+\frac{\beta_{0}}{4} \ln \left(\frac{M^{2}}{s}\right)\right] A^{c}+\left(c_{2}-\frac{\beta_{0}}{2}\right) T_{1}^{c}\right. \\
& \left.\quad+F^{c} \ln \left(\frac{M^{2}}{s}\right)+G^{c}\right\} \mathcal{D}_{0}\left(s_{4}\right) \\
& +\sigma^{B} \frac{\alpha_{s}^{2}\left(\mu_{R}^{2}\right)}{\pi^{2}} R^{(2)} \delta\left(s_{4}\right)+\frac{\alpha_{s}^{d_{\alpha_{s}}+2}\left(\mu_{R}^{2}\right)}{\pi^{2}} R_{c}^{(2)} \delta\left(s_{4}\right) .
\end{aligned}
$$

We have used the definitions

$$
\begin{gathered}
C_{D_{1}}^{(2)}=c_{3} c_{1}+c_{2}^{2}-\zeta_{2} c_{3}^{2}-\frac{\beta_{0}}{2} T_{2}+\frac{\beta_{0}}{4} c_{3} \ln \left(\frac{\mu_{R}^{2}}{M^{2}}\right)+c_{3} \frac{K}{2}-\sum_{j} \frac{\beta_{0}}{4} B_{j}^{(1)}, \\
C_{D_{0}}^{(2)}=c_{2} c_{1}-\zeta_{2} c_{3} c_{2}+\zeta_{3} c_{3}^{2}-\frac{\beta_{0}}{2} T_{1}+\frac{\beta_{0}}{4} c_{2} \ln \left(\frac{\mu_{R}^{2}}{M^{2}}\right)+d_{\alpha_{s}} \frac{\beta_{0}^{2}}{8} \ln \left(\frac{M^{2}}{s}\right)-\sum_{i} \nu_{i}^{(2)} \\
+\sum_{i} C_{i} \frac{\beta_{0}}{8}\left[\ln ^{2}\left(\frac{\mu_{F}^{2}}{M^{2}}\right)-\ln ^{2}\left(\frac{M^{2}}{s}\right)-2 \ln \left(\frac{M^{2}}{s}\right)\right]-\frac{\beta_{0}}{2} \sum_{i} \gamma_{i}^{(1)} \ln \left(\frac{M^{2}}{s}\right)
\end{gathered}
$$




$$
\begin{gathered}
-\sum_{i} C_{i} \frac{K}{2}\left[\ln \left(\frac{\mu_{F}^{2}}{M^{2}}\right)+2 \ln \left(\frac{-t_{i}}{M^{2}}\right)+\ln \left(\frac{M^{2}}{s}\right)\right]-\sum_{j}\left(B_{j}^{(2)}+\nu_{j}^{(2)}\right) \\
+\sum_{j} C_{j}\left[-\frac{\beta_{0}}{8} \ln ^{2}\left(\frac{M^{2}}{s}\right)-\frac{\beta_{0}}{4} \ln \left(\frac{M^{2}}{s}\right)-\frac{K}{2} \ln \left(\frac{M^{2}}{s}\right)\right]-\sum_{j} \frac{\beta_{0}}{2} B_{j}^{(1)} \ln \left(\frac{M^{2}}{s}\right) \\
F^{c}=\operatorname{tr}\left[H^{(0)}\left(\Gamma_{S}^{(1) \dagger}\right)^{2} S^{(0)}+H^{(0)} S^{(0)}\left(\Gamma_{S}^{(1)}\right)^{2}+2 H^{(0)} \Gamma_{S}^{(1) \dagger} S^{(0)} \Gamma_{S}^{(1)}\right] \\
G^{c}=\operatorname{tr}\left[H^{(1)} \Gamma_{S}^{(1) \dagger} S^{(0)}+H^{(1)} S^{(0)} \Gamma_{S}^{(1)}+H^{(0)} \Gamma_{S}^{(1) \dagger} S^{(1)}+H^{(0)} S^{(1)} \Gamma_{S}^{(1)}\right. \\
\left.\quad+H^{(0)} \Gamma_{S}^{(2) \dagger} S^{(0)}+H^{(0)} S^{(0)} \Gamma_{S}^{(2)}\right]
\end{gathered}
$$

In PIM kinematics simply replace $s_{4}$ by $1-z$, set $s=M^{2}$, and delete all $\ln \left(-t_{i} / M^{2}\right)$ terms.

The quantities $d_{\alpha_{s}}, \beta_{0}$, and $K$ have all been defined in Section 2 , and $\zeta_{2}=\pi^{2} / 6, \zeta_{3}=$ $1.2020569 \cdots$. Also $c_{3}, c_{2}, c_{1}, T_{1}^{c}$, and $A^{c}$ have been defined in Section 3. The virtual terms $R^{(2)}$ and $R_{c}^{(2)}$ cannot be derived from resummation. A separate calculation is needed for each process to derive those. However, all the scale-dependent terms in $R^{(2)}\left(R_{c}^{(2)}\right.$ is scale-independent) can be derived and are given explicitly in Eq. (12) of Ref. [10].

As shown in Ref. [23], the NNLO master formula passes many rigorous tests. It reproduces the NNLO soft-gluon results for all processes where these results are known. Also at NNLO the renormalization and factorization scale dependence in the physical cross section cancels out.

The master formula can in principle provide all the soft corrections at NNLO for any process. In practice, the accuracy which we can attain depends on whether the one-loop $\Gamma_{S}^{(1)}$ is known (in which case we can attain NLL accuracy; $\Gamma_{S}^{(1)}$ is known for all $2 \rightarrow 2$ processes); whether furthermore the NLO virtual terms are known (NNLL accuracy); and whether the two-loop $\Gamma_{S}^{(2)}$ is known (NNNLL accuracy). Most current results are known to NLL or NNLL accuracy. Note that $\Gamma_{S}^{(2)}$ is only known for the simplest cases of Drell-Yan and Higgs production where the color structure is trivial [23]. However, it was shown in [14] that the contributions of $\Gamma_{S}^{(2)}$ can be small so that effectively NNNLL calculations can be made in some cases even when $\Gamma_{S}^{(2)}$ is not fully known.

\section{$5 \quad$ NNNLO master formula for soft-gluon corrections}

At next-to-next-to-next-to-leading order, the expansion of Eq. (2.1), with matching to the NLO and NNLO soft-plus-virtual results, gives the NNNLO soft-gluon corrections in the $\overline{\mathrm{MS}}$ scheme and 1PI kinematics

$$
\begin{aligned}
\hat{\sigma}^{(3)} & =\sigma^{B} \frac{\alpha_{s}^{3}\left(\mu_{R}^{2}\right)}{\pi^{3}} \frac{1}{8} c_{3}^{3} \mathcal{D}_{5}\left(s_{4}\right) \\
& +\sigma^{B} \frac{\alpha_{s}^{3}\left(\mu_{R}^{2}\right)}{\pi^{3}}\left\{\frac{5}{8} c_{3}^{2} c_{2}-\frac{5}{2} c_{3} X_{3}\right\} \mathcal{D}_{4}\left(s_{4}\right)+\frac{\alpha_{s}^{d_{\alpha_{s}}+3}\left(\mu_{R}^{2}\right)}{\pi^{3}} \frac{5}{8} c_{3}^{2} A^{c} \mathcal{D}_{4}\left(s_{4}\right) \\
& +\sigma^{B} \frac{\alpha_{s}^{3}\left(\mu_{R}^{2}\right)}{\pi^{3}}\left\{c_{3} c_{2}^{2}+\frac{1}{2} c_{3}^{2} c_{1}-\zeta_{2} c_{3}^{3}+\left(\beta_{0}-4 c_{2}\right) X_{3}+2 c_{3} X_{2}-\sum_{j} C_{j} \frac{\beta_{0}^{2}}{48}\right\} \mathcal{D}_{3}\left(s_{4}\right)
\end{aligned}
$$




$$
\begin{aligned}
& +\frac{\alpha_{s}^{d_{\alpha_{s}}+3}\left(\mu_{R}^{2}\right)}{\pi^{3}}\left\{\frac{1}{2} c_{3}^{2} T_{1}^{c}+\left[2 c_{3} c_{2}-\frac{\beta_{0}}{2} c_{3}-4 X_{3}\right] A^{c}+c_{3} F^{c}\right\} \mathcal{D}_{3}\left(s_{4}\right) \\
& +\sigma^{B} \frac{\alpha_{s}^{3}\left(\mu_{R}^{2}\right)}{\pi^{3}}\left\{\frac{3}{2} c_{3} c_{2} c_{1}+\frac{1}{2} c_{2}^{3}-3 \zeta_{2} c_{3}^{2} c_{2}+\frac{5}{2} \zeta_{3} c_{3}^{3}+\left(-3 c_{1}+\frac{27}{2} \zeta_{2} c_{3}\right) X_{3}\right. \\
& +\left(3 c_{2}-\beta_{0}\right) X_{2}-\frac{3}{2} c_{3} X_{1}-\sum_{i} C_{i} \frac{\beta_{1}}{8}+\sum_{j} C_{j} \frac{\beta_{0}}{16}\left[\beta_{0} \ln \left(\frac{\mu_{R}^{2}}{M^{2}}\right)+2 K\right] \\
& \left.+\sum_{j} \frac{\beta_{0}^{2}}{16} B_{j}^{\prime(1)}+\sum_{j} \frac{3}{32} C_{j} \beta_{1}\right\} \mathcal{D}_{2}\left(s_{4}\right) \\
& +\frac{\alpha_{s}^{d_{\alpha_{s}}+3}\left(\mu_{R}^{2}\right)}{\pi^{3}}\left\{\left(\frac{3}{2} c_{3} c_{2}-3 X_{3}\right) T_{1}^{c}+\frac{3}{2}\left[c_{2}+c_{3} \ln \left(\frac{M^{2}}{s}\right)\right] F^{c}\right. \\
& +\left[\frac{3}{2} c_{2}^{2}+\frac{3}{2} c_{3} c_{1}-3 \zeta_{2} c_{3}^{2}+3 X_{2}+\frac{\beta_{0}^{2}}{4}-\frac{3}{4} \beta_{0}\left(c_{2}-\frac{c_{3}}{2} \ln \left(\frac{\mu_{R}^{2}}{M^{2}}\right)+\frac{c_{3}}{2} \ln \left(\frac{M^{2}}{s}\right)\right)\right] A^{c} \\
& \left.+\frac{3}{2} c_{3} G^{c}+\frac{1}{2} K_{3}^{c}\right\} \mathcal{D}_{2}\left(s_{4}\right) \\
& +\sigma^{B} \frac{\alpha_{s}^{3}\left(\mu_{R}^{2}\right)}{\pi^{3}}\left\{\frac{1}{2} c_{3} c_{1}^{2}+c_{2}^{2} c_{1}-\zeta_{2} c_{3}^{2} c_{1}-\frac{5}{2} \zeta_{2} c_{3} c_{2}^{2}+5 \zeta_{3} c_{3}^{2} c_{2}+\frac{5}{4} \zeta_{2}^{2} c_{3}^{3}-\frac{15}{4} \zeta_{4} c_{3}^{3}\right. \\
& -\frac{\beta_{0}^{2}}{4} \zeta_{2} c_{3}+\left(-20 \zeta_{3} c_{3}+12 \zeta_{2} c_{2}\right) X_{3}+\left(2 c_{1}-5 \zeta_{2} c_{3}\right) X_{2}+\left(\beta_{0}-2 c_{2}\right) X_{1}+c_{3} X_{0} \\
& \left.+\sum_{i} Y_{i}^{(1)}+\sum_{j} Y_{j}^{(1)}\right\} \mathcal{D}_{1}\left(s_{4}\right) \\
& +\frac{\alpha_{s}^{d_{\alpha_{s}}+3}\left(\mu_{R}^{2}\right)}{\pi^{3}}\left\{C_{D_{1}}^{(2)}\left[T_{1}^{c}-A^{c} \ln \left(\frac{M^{2}}{s}\right)\right]+c_{3} \operatorname{tr}\left[H^{(2)} S^{(0)}+H^{(0)} S^{(2)}+H^{(1)} S^{(1)}\right]\right. \\
& +\left[2 c_{2} c_{1}-5 \zeta_{2} c_{3} c_{2}+5 \zeta_{3} c_{3}^{2}+12 \zeta_{2} X_{3}-2 X_{1}+\ln \left(\frac{M^{2}}{s}\right)\left(c_{2}^{2}+c_{3} c_{1}-\zeta_{2} c_{3}^{2}+2 X_{2}\right)\right. \\
& -\frac{\beta_{0}}{2}\left(c_{1}-\frac{5}{2} \zeta_{2} c_{3}-c_{2} \ln \left(\frac{\mu_{R}^{2}}{M^{2}}\right)-\frac{c_{3}}{2} \ln \left(\frac{M^{2}}{s}\right) \ln \left(\frac{\mu_{R}^{2}}{M^{2}}\right)+c_{2} \ln \left(\frac{M^{2}}{s}\right)\right) \\
& \left.+\frac{\beta_{0}^{2}}{4}\left(\ln \left(\frac{M^{2}}{s}\right)-\ln \left(\frac{\mu_{R}^{2}}{M^{2}}\right)\right)-\frac{\beta_{1}}{8}\right] A^{c} \\
& +\left[-\frac{5}{2} \zeta_{2} c_{3}+c_{1}+2 c_{2} \ln \left(\frac{M^{2}}{s}\right)+\frac{c_{3}}{2} \ln ^{2}\left(\frac{M^{2}}{s}\right)-\frac{\beta_{0}}{2}\right] F^{c} \\
& \left.+\left[c_{3} \ln \left(\frac{M^{2}}{s}\right)+2 c_{2}\right] G^{c}-\beta_{0}\left(G^{c}-M^{c}\right)+K_{2}^{c}+K_{3}^{c} \ln \left(\frac{M^{2}}{s}\right)\right\} \mathcal{D}_{1}\left(s_{4}\right) \\
& +\sigma^{B} \frac{\alpha_{s}^{3}\left(\mu_{R}^{2}\right)}{\pi^{3}}\left\{\frac{1}{2} c_{2} c_{1}^{2}+3 \zeta_{5} c_{3}^{3}-\frac{15}{4} \zeta_{4} c_{3}^{2} c_{2}-2 \zeta_{2} \zeta_{3} c_{3}^{3}+\zeta_{3} c_{3}^{2} c_{1}+2 \zeta_{3} c_{3} c_{2}^{2}+\frac{5}{4} \zeta_{2}^{2} c_{3}^{2} c_{2}\right. \\
& -\zeta_{2} c_{3} c_{2} c_{1}-\frac{1}{2} \zeta_{2} c_{2}^{3}+\left(15 \zeta_{4} c_{3}-8 \zeta_{3} c_{2}-6 \zeta_{2}^{2} c_{3}+3 \zeta_{2} c_{1}\right) X_{3}+\left(4 \zeta_{3} c_{3}-3 \zeta_{2} c_{2}\right) X_{2} \\
& +\left(\zeta_{2} c_{3}-c_{1}\right) X_{1}+c_{2} X_{0}-\frac{\beta_{0}^{2}}{4} T_{1} \ln \left(\frac{\mu_{R}^{2}}{M^{2}}\right)+\frac{\beta_{0}^{2}}{16} T_{2} \ln ^{2}\left(\frac{\mu_{R}^{2}}{M^{2}}\right)-\frac{\beta_{0}^{2}}{4} T_{1} \ln \left(\frac{M^{2}}{s}\right)
\end{aligned}
$$




$$
\left.\begin{array}{rl}
- & \left.\frac{\beta_{1}}{8} T_{1}+\sum_{i} Y_{i}^{(0)}+\sum_{j} Y_{j}^{(0)}\right\} \mathcal{D}_{0}\left(s_{4}\right) \\
+ & \frac{\alpha_{s}^{d_{\alpha_{s}}+3}\left(\mu_{R}^{2}\right)}{\pi^{3}}\left\{C_{D_{0}}^{(2)}\left[T_{1}^{c}-A^{c} \ln \left(\frac{M^{2}}{s}\right)\right]+\left[c_{2} \ln \left(\frac{M^{2}}{s}\right)-\zeta_{2} c_{3}+c_{1}\right] G^{c}\right. \\
+ & {\left[\frac{1}{2}\left(c_{1}+\frac{\zeta_{2}}{2} c_{3}\right)^{2}+\frac{9}{8} \zeta_{2}^{2} c_{3}^{2}-\frac{15}{4} \zeta_{4} c_{3}^{2}+4 \zeta_{3} c_{3} c_{2}-\frac{3}{2} \zeta_{2} c_{3} c_{1}-\frac{3}{2} \zeta_{2} c_{2}^{2}-8 \zeta_{3} X_{3}\right.} \\
& \left.-3 \zeta_{2} X_{2}+X_{0}+\left(c_{2} c_{1}-\zeta_{2} c_{3} c_{2}+\zeta_{3} c_{3}^{2}+3 \zeta_{2} X_{3}-X_{1}\right) \ln \left(\frac{M^{2}}{s}\right)\right] A^{c} \\
- & \frac{\beta_{0}}{2}\left[-\frac{c_{2}}{2} \ln \left(\frac{M^{2}}{s}\right) \ln \left(\frac{\mu_{R}^{2}}{M^{2}}\right)+2 \zeta_{3} c_{3}-\frac{3}{2} \zeta_{2} c_{2}\right. \\
+ & {\left[\frac{c_{2}}{2} \ln ^{2}\left(\frac{M^{2}}{s}\right)+\left(c_{1}-\zeta_{2} c_{3}\right)\right.} \\
+ & \left.\left.\left[\frac{\beta_{0}^{2}}{16}\left(-2 \ln \left(\frac{\mu_{R}^{2}}{M^{2}}\right)+\ln \left(\frac{M^{2}}{s}\right)\right)\right] A^{c}-\frac{\beta_{0}}{4}\right) \ln \left(\frac{M^{2}}{s}\right)+2 \zeta_{3} c_{3}-\frac{3}{2} \zeta_{2} c_{2}+\frac{\beta_{0}}{4} \ln \left(\frac{\mu_{R}^{2}}{M^{2}}\right)\right] F^{c} \\
& \left.-\frac{\beta_{1}^{2}}{16}\left(\ln \left(\frac{M^{2}}{s}\right)-\ln \left(\frac{\mu_{R}^{2}}{M^{2}}\right)\right)\right] M^{c}+c_{2} \operatorname{tr}\left[H^{(2)} S^{(0)}+H^{(0)} S^{(2)}+H^{(1)} S^{(1)}\right] \\
+ & \left.K_{1}^{c}+K_{2}^{c} \ln \left(\frac{M^{2}}{s}\right)+\frac{1}{2}\left[-\zeta_{2}+\ln ^{2}\left(\frac{M^{2}}{s}\right)\right] K_{3}^{c}\right\} \mathcal{D}_{0}\left(s_{4}\right) . \\
s
\end{array}\right)
$$

The quantities $d_{\alpha_{s}}, \beta_{0}$, and $K$ have been defined in Section 2, while $c_{3}, c_{2}, c_{1}, T_{1}^{c}$, and $A^{c}$ have been defined in Section 3. Also $C_{D_{1}}^{(2)}, C_{D_{0}}^{(2)}, F^{c}, G^{c}, \zeta_{2}$, and $\zeta_{3}$ have been defined in Section 4, and $\zeta_{4}=\pi^{4} / 90$. The expressions for $\beta_{1}, X_{3}, X_{2}, X_{1}, X_{0}, Y_{i}^{(1)}, Y_{j}^{(1)}, Y_{i}^{(0)}, Y_{j}^{(0)}, M^{c}, K_{1}^{c}, K_{2}^{c}$, and $K_{3}^{c}$ are given in the Appendix.

Note again that in PIM kinematics we simply replace $s_{4}$ by $1-z$, set $s=M^{2}$, and drop the terms with $\ln \left(-t_{i} / M^{2}\right)$ in the above formula and in all the expressions given in the Appendix.

This NNNLO master equation gives the structure of the NNNLO soft corrections and can provide the full soft corrections explicitly if all the two-loop and three-loop quantities are known. Therefore for processes with non-trivial color structure we are currently limited to NLL or NNLL accuracy, as the applications to charged Higgs and top quark production in the next two sections illustrate. The structure of the corrections as presented here can be useful for checking future calculations if and when such three-loop quantities become available. Also note that scale logarithms and $\zeta_{i}$ constants will be kept as appropriate at subleading logs as explained in the next two sections.

When the color structure of the hard scattering is simple, i.e. when $H, S$, and $\Gamma_{S}$ are simply $1 \times 1$ matrices, then the above expressions can be simplified. We can then easily absorb $A^{c}$ into $c_{2}$ in Eq. (3.1), and $T_{1}^{c}$ into $c_{1}$, by redefining $c_{2}$ and $c_{1}$. Then all the terms are proportional to $\sigma^{B}$ in Eqs. (3.1), (4.1), and (5.1). We will see this explicitly in the two applications in the next two sections. 


\section{$6 \quad$ Charged Higgs production via $b g \rightarrow t H^{-}$}

Charged Higgs production is a process of great interest at the LHC. The charged Higgs boson, if discovered, would be an unmistakable sign of new physics beyond the Standard Model [21]. A promising channel of discovery is associated production with a top quark via bottom-gluon fusion for which SUSY and QCD radiative corrections have been calculated [30, 31, 32, 33. NLO and NNLO soft-gluon corrections to this process were recently studied in [18] where the corrections were found to be large, especially for a very massive charged Higgs.

We now apply our NNNLO master formula to charged Higgs production in the Minimal Supersymmetric Standard Model (MSSM) via bottom gluon fusion, a process with simple color flow, at NLL accuracy. We study the process $b\left(p_{b}\right)+g\left(p_{g}\right) \rightarrow t\left(p_{t}\right)+H^{-}\left(p_{H^{-}}\right)$in $1 \mathrm{PI}$ kinematics and define the kinematical invariants $s=\left(p_{b}+p_{g}\right)^{2}, t=\left(p_{b}-p_{t}\right)^{2}, u=\left(p_{g}-p_{t}\right)^{2}$, and $s_{4}=s+t+u-m_{t}^{2}-m_{H^{-}}^{2}$, where $m_{t}$ is the top quark mass and $m_{H^{-}}$is the charged Higgs mass (we take the bottom quark $m_{b}=0$ in the kinematics [18]).

The NLO coefficients of Section 3 here take the values $c_{3}=2\left(C_{F}+C_{A}\right)$ and $c_{2}=c_{2}^{\mu}+T_{2}$ with $c_{2}^{\mu}=-\left(C_{F}+C_{A}\right) \ln \left(\mu_{F}^{2} / m_{H^{-}}^{2}\right)$ and

$$
\begin{aligned}
T_{2}= & 2 \operatorname{Re} \Gamma_{S}^{(1)}-C_{F}-C_{A}-2 C_{F} \ln \left(\frac{-u+m_{H^{-}}^{2}}{m_{H^{-}}^{2}}\right)-2 C_{A} \ln \left(\frac{-t+m_{H^{-}}^{2}}{m_{H^{-}}^{2}}\right) \\
& -\left(C_{F}+C_{A}\right) \ln \left(\frac{m_{H^{-}}^{2}}{s}\right)
\end{aligned}
$$

where $\operatorname{Re} \Gamma_{S}^{(1)}$ denotes the real part of the one-loop soft anomalous dimension [18],

$$
\Gamma_{S}^{(1)}=C_{F} \ln \left(\frac{-t+m_{t}^{2}}{m_{t} \sqrt{s}}\right)+\frac{C_{A}}{2} \ln \left(\frac{-u+m_{t}^{2}}{-t+m_{t}^{2}}\right)+\frac{C_{A}}{2}(1-\pi i) .
$$

Note that, due to the simple color structure of this process, $\Gamma_{S}$ is simply a $1 \times 1$ matrix. Here, as described in the last paragraph of the previous section, we have aborbed the term $2 \operatorname{Re} \Gamma_{S}^{(1)}$, which arises from $A^{c}$, into $T_{2}$. Also,

$$
\begin{aligned}
c_{1}^{\mu}= & {\left[C_{F} \ln \left(\frac{-u+m_{H^{-}}^{2}}{m_{H^{-}}^{2}}\right)+C_{A} \ln \left(\frac{-t+m_{H^{-}}^{2}}{m_{H^{-}}^{2}}\right)-\frac{3}{4} C_{F}-\frac{\beta_{0}}{4}\right] \ln \left(\frac{\mu_{F}^{2}}{m_{H^{-}}^{2}}\right) } \\
& +\frac{\beta_{0}}{4} \ln \left(\frac{\mu_{R}^{2}}{m_{H^{-}}^{2}}\right) .
\end{aligned}
$$

The various terms from the Appendix used in the NNNLO corrections here take the values $X_{3}=\beta_{0} c_{3} / 12, X_{2}^{\mu}=\left(\beta_{0} / 8\right) c_{3} \ln \left(\mu_{R}^{2} / m_{H^{-}}^{2}\right)$,

$$
\begin{gathered}
X_{1}^{\mu^{2}}=-\frac{\beta_{0}}{4} c_{2}^{\mu} \ln \left(\frac{\mu_{R}^{2}}{m_{H^{-}}^{2}}\right)-\left(C_{F}+C_{A}\right) \frac{\beta_{0}}{8} \ln ^{2}\left(\frac{\mu_{F}^{2}}{m_{H^{-}}^{2}}\right), \\
X_{1}^{\zeta}=\frac{\beta_{0}}{4} \zeta_{2} c_{3},
\end{gathered}
$$

where $X_{2}^{\mu}$ denotes the scale logarithm terms in $X_{2}, X_{1}^{\mu^{2}}$ denotes terms involving squares of the scale logarithms in $X_{1}$, and $X_{1}^{\zeta}$ denotes the $\zeta_{i}$ terms in $X_{1}$. 


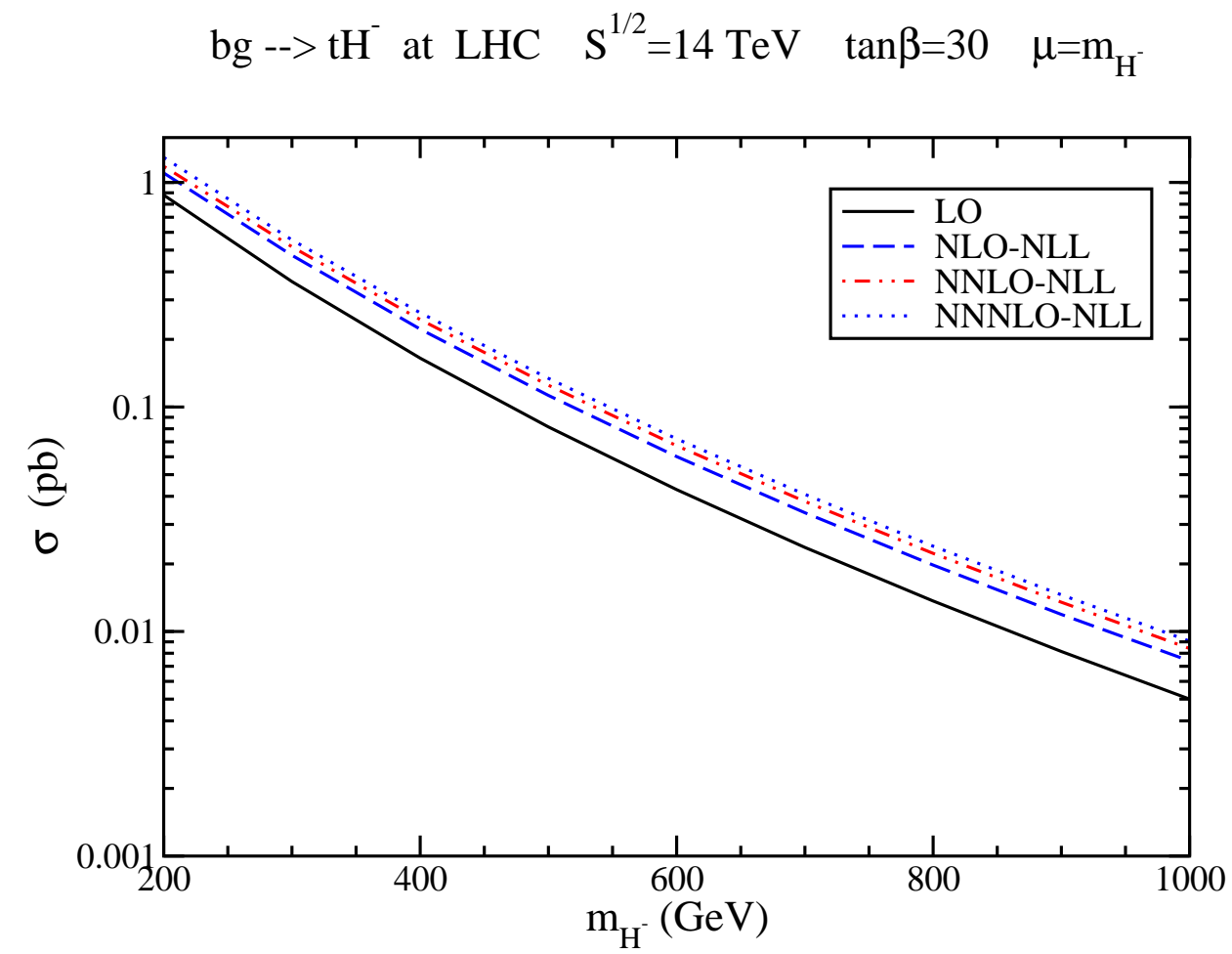

Figure 1: The total cross section for charged Higgs production at the LHC.

The NNNLO-NLL corrections are then given by

$$
\begin{aligned}
\hat{\sigma}^{(3)} & =\sigma^{B} \frac{\alpha_{s}^{3}\left(\mu_{R}^{2}\right)}{\pi^{3}}\left\{\frac{1}{8} c_{3}^{3} \mathcal{D}_{5}\left(s_{4}\right)+\left[\frac{5}{8} c_{3}^{2} c_{2}-\frac{5}{2} c_{3} X_{3}\right] \mathcal{D}_{4}\left(s_{4}\right)\right. \\
+ & {\left[c_{3}\left(c_{2}^{\mu}\right)^{2}+2 c_{3} T_{2} c_{2}^{\mu}+\frac{1}{2} c_{3}^{2} c_{1}^{\mu}-\zeta_{2} c_{3}^{3}-4 c_{2}^{\mu} X_{3}+2 c_{3} X_{2}^{\mu}\right] \mathcal{D}_{3}\left(s_{4}\right) } \\
+ & {\left[\frac{3}{2} c_{3} c_{2}^{\mu} c_{1}^{\mu}+\frac{1}{2}\left(c_{2}^{\mu}\right)^{3}+\frac{3}{2} T_{2}\left(c_{2}^{\mu}\right)^{2}-3 \zeta_{2} c_{3}^{2} c_{2}+\frac{5}{2} \zeta_{3} c_{3}^{3}\right.} \\
& \left.+\frac{27}{2} \zeta_{2} c_{3} X_{3}+3 c_{2}^{\mu} X_{2}^{\mu}-\frac{3}{2} c_{3}\left(X_{1}^{\mu^{2}}+X_{1}^{\zeta}\right)\right] \mathcal{D}_{2}\left(s_{4}\right) \\
+ & {\left[\left(c_{2}^{\mu}\right)^{2} c_{1}^{\mu}-\zeta_{2} c_{3}^{2} c_{1}^{\mu}-\frac{5}{2} \zeta_{2} c_{3}\left(\left(c_{2}^{\mu}\right)^{2}+2 T_{2} c_{2}^{\mu}\right)+5 \zeta_{3} c_{3}^{2} c_{2}^{\mu}\right.} \\
& \left.\left.+12 \zeta_{2} c_{2}^{\mu} X_{3}-5 \zeta_{2} c_{3} X_{2}^{\mu}-2 c_{2}^{\mu}\left(X_{1}^{\mu^{2}}+X_{1}^{\zeta}\right)\right] \mathcal{D}_{1}\left(s_{4}\right)\right\} .
\end{aligned}
$$

Because we absorbed $A^{c}$ into $c_{2}$ the corrections take a simple form, simply multiplying the Born term $\sigma^{B}$. Note that consistent with a NLL calculation we include all $\mathcal{D}_{5}$ (LL) and $\mathcal{D}_{4}$ (NLL) terms. In addition, we calculate all scale logarithms at NLL accuracy. This means that for coefficients of $\ln ^{i}\left(\mu^{2} / m^{2}\right)$ we include the most singular plus distribution and the next-mostsingular one [13]. Thus, we also include all scale logarithms in the $\mathcal{D}_{3}$ terms, the cubed and squared scale logarithms in the $\mathcal{D}_{2}$ terms, and the cubed scale logarithms in the $\mathcal{D}_{1}$ terms. With respect to the subleading $\zeta_{i}$ terms that arise from inversion from moment to momentum 


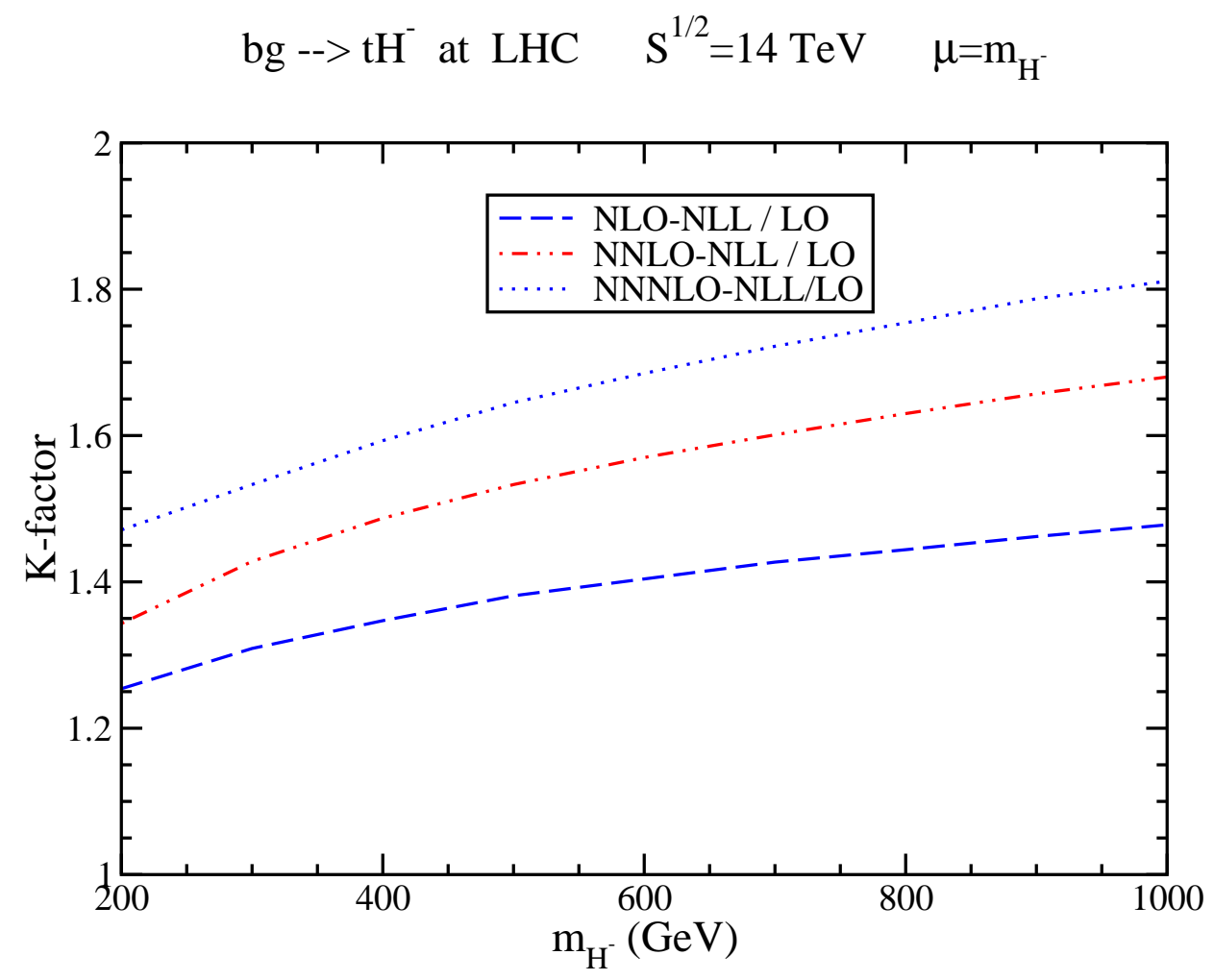

Figure 2: The $K$-factors for charged Higgs production at the LHC.

space, we include only those that we can calculate exactly (for a discussion of the numerical effects of such terms see Ref. [9]). Thus we include all $\zeta_{i}$ terms in the $\mathcal{D}_{3}$ and $\mathcal{D}_{2}$ terms, and all $\zeta_{i}$ terms multiplying scale logarithms in the $\mathcal{D}_{1}$ term.

In Fig. 1 we plot the cross section versus charged Higgs mass for $p p$ collisions at the LHC with $\sqrt{S}=14 \mathrm{TeV}$ using the MRST2002 approximate NNLO parton distribution functions 34 with the respective three-loop evaluation of $\alpha_{s}$. We set the factorization scale equal to the renormalization scale and denote this common scale by $\mu$. We show results for the LO, NLONLL, NNLO-NLL, and NNNLO-NLL cross sections, all with a choice of scale $\mu=m_{H^{-}}$. In the calculation we choose a value $\tan \beta=30$; here $\tan \beta$ is the ratio of the vacuum expectation values of the two Higgs doublets in the MSSM. It is straightforward to calculate results for any other value of $\tan \beta$, since the only dependence on $\beta$ is in a factor $m_{b}^{2} \tan ^{2} \beta+m_{t}^{2} \cot ^{2} \beta$ appearing in the Born term. The cross sections span over two orders of magnitude in the mass range shown, $200 \mathrm{GeV} \leq m_{H^{-}} \leq 1000 \mathrm{GeV}$. The NLO, NNLO, and NNNLO threshold corrections are positive and provide a significant enhancement to the lowest-order result. We note that the cross sections for the related process $\bar{b} g \rightarrow \bar{t} H^{+}$in the MSSM are exactly the same.

In Fig. 2 we plot the $K$-factors, i.e. ratios of cross sections at higher orders to the LO result, to better show the relative size of the corrections. The NLO-NLL / LO curve shows that the NLO soft-gluon corrections enhance the LO cross section by approximately $25 \%$ to $50 \%$ depending on the mass of the charged Higgs. As expected the corrections increase for 
higher charged Higgs masses as we get closer to threshold. The NNLO-NLL / LO curve shows that if we include the NNLO threshold corrections we get an enhancement over the LO result of approximately $35 \%$ to $70 \%$ in the range of masses shown. Again the enhancement increases with charged Higgs mass, as expected. Finally, the NNNLO-NLL / LO curve shows the further enhancement that the NNNLO soft-gluon corrections provide, approximately $45 \%$ to $80 \%$ over LO. We note that the NNNLO soft-gluon corrections are quite significant in this case. This is not necessarily typical for other processes, but happens here because of the very massive final state. Another process where NNNLO soft-gluon corrections are known to be big is inclusive hadron production at high transverse momentum [35].

\section{$7 \quad$ Top quark production via $q \bar{q} \rightarrow t \bar{t}$}

The study of the top quark is important in understanding the electroweak sector and searching for new physics. The top quark is now being actively studied at Run II at the Tevatron [36, 37, 38. Theoretically, the production cross section has been studied to NNLO and NNNLL in both 1PI and PIM kinematics [14]. The corrections are moderate and they substantially decrease the scale dependence of the cross section. Transverse momentum distributions are also known to NNLO-NNNLL [14, while rapidity distributions have been presented in Ref. 39 .

Here we apply our NNNLO master formula to top quark pair production via quark-antiquark annihilation, which is the dominant partonic subprocess at the Tevatron. We study the channel $q\left(p_{a}\right)+\bar{q}\left(p_{b}\right) \rightarrow t\left(p_{1}\right)+\bar{t}\left(p_{2}\right)$ in 1PI kinematics, and define the kinematical invariants $s=$ $\left(p_{a}+p_{b}\right)^{2}, t_{1}=\left(p_{b}-p_{1}\right)^{2}-m_{t}^{2}, u_{1}=\left(p_{a}-p_{1}\right)^{2}-m_{t}^{2}$, and $s_{4}=s+t_{1}+u_{1}$, with $m_{t}$ the top quark mass. For this process, which has complex color flow, $H, S$, and $\Gamma_{S}$ are $2 \times 2$ matrices. However, $H^{(0)}$ has a particularly simple form in a singlet-octet color basis (the only non-zero element is $H_{22}^{(0)}$ [9, 13]) so that simplifications arise.

The NLO coefficients of Section 3 here take the values $c_{3}=4 C_{F}$ and $c_{2}=c_{2}^{\mu}+T_{2}$, with $c_{2}^{\mu}=-2 C_{F} \ln \left(\mu_{F}^{2} / m_{t}^{2}\right)$ and

$$
T_{2}=2 \operatorname{Re} \Gamma_{S, 22}^{(1)}-2 C_{F}-2 C_{F} \ln \left(\frac{t_{1} u_{1}}{m_{t}^{4}}\right)-2 C_{F} \ln \left(\frac{m_{t}^{2}}{s}\right),
$$

where $\operatorname{Re} \Gamma_{S, 22}^{(1)}$ denotes the real part of the 22 element of the soft anomalous dimension matrix [5, 9],

$$
\Gamma_{S, 22}^{(1)}=C_{F}\left[4 \ln \left(\frac{u_{1}}{t_{1}}\right)-L_{\beta}-i \pi\right]+\frac{C_{A}}{2}\left[-3 \ln \left(\frac{u_{1}}{t_{1}}\right)-\ln \left(\frac{m_{t}^{2} s}{t_{1} u_{1}}\right)+L_{\beta}+i \pi\right]
$$

with $L_{\beta}=\left[\left(1-2 m_{t}^{2} / s\right) / \beta\right][\ln ((1-\beta) /(1+\beta))+i \pi]$ and $\beta=\sqrt{1-4 m_{t}^{2} / s}$. Note that even though we have nontrivial color matrices for this process, due to the simple form of the $H$ matrix here we have aborbed the term $2 \operatorname{Re} \Gamma_{S, 22}^{(1)}$, which arises from $A^{c}$, into $T_{2}$. To be precise, $A^{c}=\left(\sigma^{B} / \alpha_{s}^{2}\right) 2 \operatorname{Re} \Gamma_{S, 22}^{(1)}$. Also, $c_{1}=c_{1}^{\mu}+T_{1}$ with

$$
c_{1}^{\mu}=C_{F}\left[\ln \left(\frac{t_{1} u_{1}}{m_{t}^{4}}\right)-\frac{3}{2}\right] \ln \left(\frac{\mu_{F}^{2}}{m_{t}^{2}}\right)+\frac{\beta_{0}}{2} \ln \left(\frac{\mu_{R}^{2}}{m_{t}^{2}}\right)
$$




\section{$\mathrm{q} \overline{\mathrm{q}}-->\mathrm{tt}$ at the Tevatron $\mathrm{S}^{1 / 2}=1.96 \mathrm{TeV} \quad \mathrm{m}_{\mathrm{t}}=175 \mathrm{GeV}$}

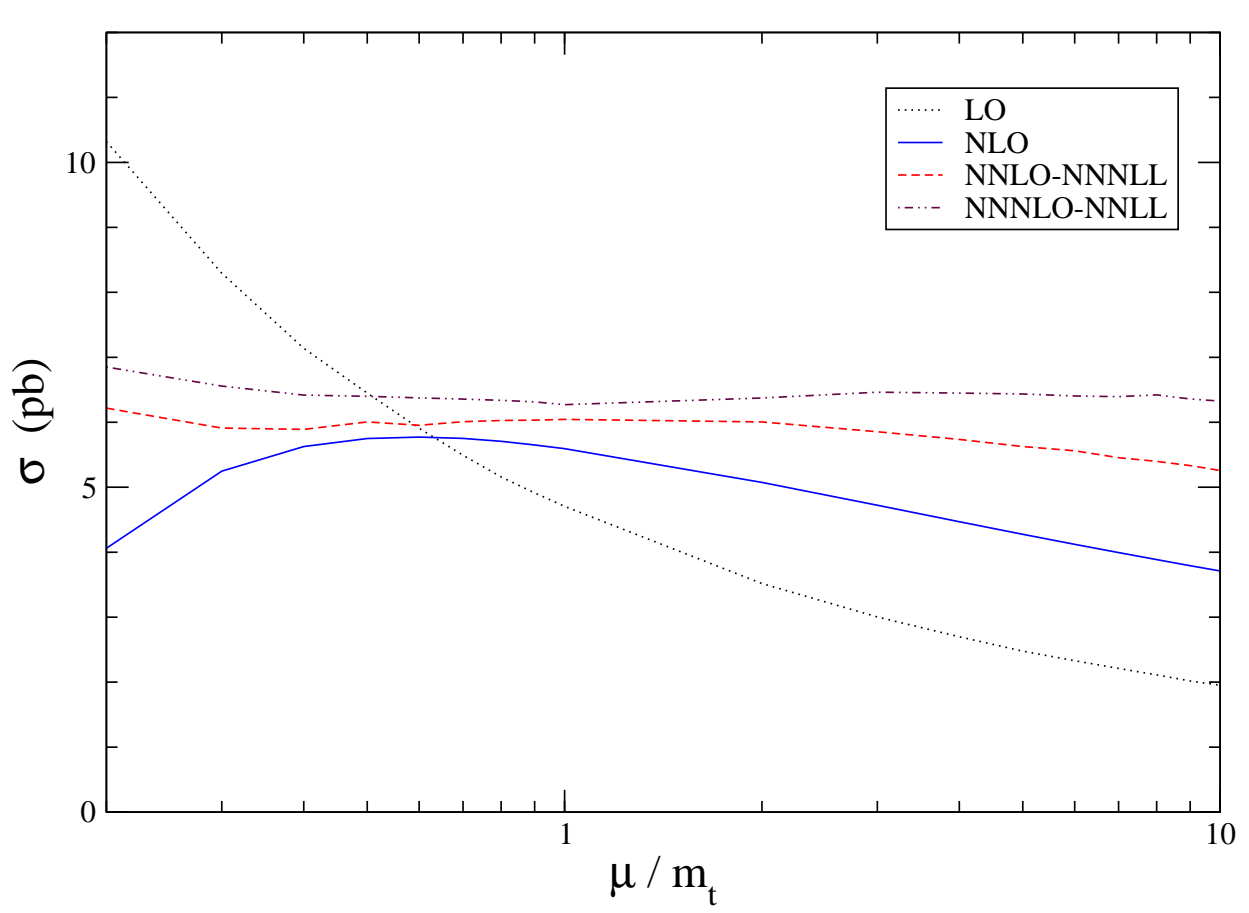

Figure 3: The scale dependence of top production in the $q \bar{q}$ channel at the Tevatron.

and $T_{1}$ as given by the $\delta\left(s_{4}\right)$ terms in Eq. (4.7) of Ref. [40] (we have effectively absorbed $T_{1}^{c}$ into $c_{1}$; for details see [14]). Also, $F^{c}=\left(\sigma^{B} / \alpha_{s}^{2}\right)\left[4\left(\operatorname{Re}_{S, 22}^{(1)}\right)^{2}+4 \Gamma_{S, 12}^{(1)} \Gamma_{S, 21}^{(1)}\right]$ with $\Gamma_{S, 12}^{(1)}=$ $\left(C_{F} / C_{A}\right) \ln \left(u_{1} / t_{1}\right)$ and $\Gamma_{S, 21}^{(1)}=2 \ln \left(u_{1} / t_{1}\right)$ the 12 and 21 elements of the soft anomalous dimension matrix.

The various terms from the Appendix used in the NNNLO corrections here take the values $X_{3}=\beta_{0} c_{3} / 12, X_{2}=-\left(\beta_{0} / 4\right) T_{2}+\left(\beta_{0} / 8\right) c_{3} \ln \left(\mu_{R}^{2} / m_{t}^{2}\right)+c_{3} K / 4, X_{2}^{\mu}=\left(\beta_{0} / 8\right) c_{3} \ln \left(\mu_{R}^{2} / m_{t}^{2}\right)$,

$$
\begin{gathered}
X_{1}^{\mu^{2}}=-\frac{\beta_{0}}{4} c_{2}^{\mu} \ln \left(\frac{\mu_{R}^{2}}{m_{t}^{2}}\right)-C_{F} \frac{\beta_{0}}{4} \ln ^{2}\left(\frac{\mu_{F}^{2}}{m_{t}^{2}}\right), \\
X_{1}^{\mu}=-\frac{\beta_{0}}{4} T_{2} \ln \left(\frac{\mu_{R}^{2}}{m_{t}^{2}}\right)+C_{F} K \ln \left(\frac{\mu_{F}^{2}}{m_{t}^{2}}\right), \\
X_{1}^{\zeta}=\frac{\beta_{0}}{4} \zeta_{2} c_{3}, \\
X_{0}^{\mu^{2}}=\frac{\beta_{0}}{4} c_{1}^{\mu} \ln \left(\frac{\mu_{R}^{2}}{m_{t}^{2}}\right)-\frac{\beta_{0}^{2}}{16} \ln ^{2}\left(\frac{\mu_{R}^{2}}{m_{t}^{2}}\right) \\
+\frac{\beta_{0}}{8}\left[\frac{3}{2} C_{F}-C_{F} \ln \left(\frac{t_{1} u_{1}}{m_{t}^{4}}\right)\right] \ln ^{2}\left(\frac{\mu_{F}^{2}}{m_{t}^{2}}\right),
\end{gathered}
$$




$$
\begin{gathered}
X_{0}^{\zeta}=\frac{\beta_{0}}{6} \zeta_{3} c_{3}-\frac{\beta_{0}}{4} \zeta_{2} T_{2}+\frac{\beta_{0}}{8} \zeta_{2} c_{3} \ln \left(\frac{\mu_{R}^{2}}{m_{t}^{2}}\right)+C_{F} \zeta_{2} K, \\
Y_{q}^{\left(1, \mu^{2}\right)}=C_{F} \frac{\beta_{0}^{2}}{8} \ln ^{2}\left(\frac{\mu_{F}^{2}}{\mu_{R}^{2}}\right) \\
Y_{q}^{\left(0, \mu^{3}\right)}=-C_{F} \frac{\beta_{0}^{2}}{48}\left[\ln ^{3}\left(\frac{\mu_{F}^{2}}{\mu_{R}^{2}}\right)+\ln ^{3}\left(\frac{\mu_{R}^{2}}{m_{t}^{2}}\right)\right],
\end{gathered}
$$

where $X_{i}^{\mu^{n}}$ denotes the terms with scale logarithms to the $n$-th power in $X_{i}, X_{0}^{\zeta}$ denotes the $\zeta_{i}$ terms in $X_{0}, Y_{q}^{\left(1, \mu^{2}\right)}$ denotes terms involving squares of the scale logarithms in $Y_{q}^{(1)}$, and $Y_{q}^{\left(0, \mu^{3}\right)}$ denotes terms involving cubes of the scale logarithms in $Y_{q}^{(0)}$.

The NNNLO-NNLL corrections are then given by

$$
\begin{aligned}
\hat{\sigma}^{(3)}= & \sigma^{B} \frac{\alpha_{s}^{3}\left(\mu_{R}^{2}\right)}{\pi^{3}}\left\{\frac{1}{8} c_{3}^{3} \mathcal{D}_{5}\left(s_{4}\right)+\left[\frac{5}{8} c_{3}^{2} c_{2}-\frac{5}{2} c_{3} X_{3}\right] \mathcal{D}_{4}\left(s_{4}\right)\right. \\
+ & {\left[c_{3} c_{2}^{2}+\frac{1}{2} c_{3}^{2} c_{1}-\zeta_{2} c_{3}^{3}+\left(\beta_{0}-4 c_{2}\right) X_{3}+2 c_{3} X_{2}+4 c_{3} \Gamma_{S, 12}^{(1)} \Gamma_{S, 21}^{(1)}\right] \mathcal{D}_{3}\left(s_{4}\right) } \\
+ & {\left[\frac{3}{2} c_{3} c_{2}^{\mu} c_{1}+\frac{3}{2} c_{3} T_{2} c_{1}^{\mu}+\frac{1}{2}\left(c_{2}^{3}-T_{2}^{3}\right)-3 \zeta_{2} c_{3}^{2} c_{2}+\frac{5}{2} \zeta_{3} c_{3}^{3}-3 c_{1}^{\mu} X_{3}+\frac{27}{2} \zeta_{2} c_{3} X_{3}\right.} \\
& \left.+\left(3 T_{2}-\beta_{0}\right) X_{2}^{\mu}+3 c_{2}^{\mu} X_{2}-\frac{3}{2} c_{3}\left(X_{1}^{\mu}+X_{1}^{\mu^{2}}+X_{1}^{\zeta}\right)+6 c_{2}^{\mu} \Gamma_{S, 12}^{(1)} \Gamma_{S, 21}^{(1)}\right] \mathcal{D}_{2}\left(s_{4}\right) \\
+ & {\left[\frac{1}{2} c_{3}\left(c_{1}^{\mu}\right)^{2}+\left(c_{2}^{\mu}\right)^{2} c_{1}+2 T_{2} c_{2}^{\mu} c_{1}^{\mu}-\zeta_{2} c_{3}^{2} c_{1}-\frac{5}{2} \zeta_{2} c_{3} c_{2}^{2}+5 \zeta_{3} c_{3}^{2} c_{2}+\frac{5}{4} \zeta_{2}^{2} c_{3}^{3}-\frac{15}{4} \zeta_{4} c_{3}^{3}\right.} \\
& -\frac{\beta_{0}^{2}}{4} \zeta_{2} c_{3}+\left(-20 \zeta_{3} c_{3}+12 \zeta_{2} c_{2}\right) X_{3}+2 c_{1}^{\mu} X_{2}^{\mu}-5 \zeta_{2} c_{3} X_{2}+\left(\beta_{0}-2 c_{2}\right)\left(X_{1}^{\mu^{2}}+X_{1}^{\zeta}\right) \\
& \left.-2 c_{2}^{\mu} X_{1}^{\mu}+c_{3}\left(X_{0}^{\mu^{2}}+X_{0}^{\zeta}\right)+2 Y_{q}^{\left(1, \mu^{2}\right)}-10 \zeta_{2} c_{3} \Gamma_{S, 12}^{(1)} \Gamma_{S, 21}^{(1)}\right] \mathcal{D}_{1}\left(s_{4}\right) \\
+ & {\left[\frac{1}{2} c_{2}^{\mu}\left(c_{1}^{\mu}\right)^{2}-\frac{15}{4} \zeta_{4} c_{3}^{2} c_{2}^{\mu}+\zeta_{3} c_{3}^{2} c_{1}^{\mu}+2 \zeta_{3} c_{3}\left(c_{2}^{2}-T_{2}^{2}\right)+\frac{5}{4} \zeta_{2}^{2} c_{3}^{2} c_{2}^{\mu}-\zeta_{2} c_{3} c_{2} c_{1}^{\mu}\right.} \\
& -\zeta_{2} c_{3} c_{2}^{\mu} T_{1}-\frac{1}{2} \zeta_{2}\left(c_{2}^{3}-T_{2}^{3}\right)+\left(-8 \zeta_{3} c_{2}^{\mu}+3 \zeta_{2} c_{1}^{\mu}\right) X_{3}+\left(4 \zeta_{3} c_{3}-3 \zeta_{2} T_{2}\right) X_{2}^{\mu} \\
& -3 \zeta_{2} c_{2}^{\mu} X_{2}+\zeta_{2} c_{3}\left(X_{1}^{\mu}+X_{1}^{\mu^{2}}\right)-c_{1}^{\mu}\left(X_{1}^{\mu^{2}}+X_{1}^{\zeta}\right)+c_{2}^{\mu}\left(X_{0}^{\mu^{2}}+X_{0}^{\zeta}\right)+2 Y_{q}^{\left(0, \mu^{3}\right)} \\
& \left.\left.-6 \zeta_{2} c_{2}^{\mu} \Gamma_{S, 12}^{(1)} \Gamma_{S, 21}^{(1)}\right] \mathcal{D}_{0}\left(s_{4}\right)\right\} .
\end{aligned}
$$

Because we have absorbed $A^{c}$ into $c_{2}$, and $T_{1}^{c}$ into $c_{1}$, the corrections take a simple form, simply multiplying the LO term $\sigma^{B}$. Note that consistent with a NNLL calculation we include all $\mathcal{D}_{5}, \mathcal{D}_{4}$, and $\mathcal{D}_{3}$ terms. In addition, we calculate all scale logarithms at NNLL accuracy. This means that for coefficients of $\ln ^{i}\left(\mu^{2} / m^{2}\right)$ we include the most singular plus distribution and the two next-most-singular ones [13. So we also include all scale logarithms in the $\mathcal{D}_{2}$ terms, the cubed and squared scale logarithms in the $\mathcal{D}_{1}$ terms, and the cubed scale logarithms in the $\mathcal{D}_{0}$ terms. With respect to the subleading $\zeta_{i}$ terms that arise from inversion from moment to momentum space, we include only those that we can calculate exactly (see Ref. [9]). Thus we include all $\zeta_{i}$ terms in the $\mathcal{D}_{2}$ and $\mathcal{D}_{1}$ terms, and all $\zeta_{i}$ terms multiplying scale logarithms in the $\mathcal{D}_{0}$ term. 
In Fig. [3] we plot the scale dependence of the cross section for $q \bar{q} \rightarrow t \bar{t}$ at the Tevatron Run II for a top quark mass $m_{t}=175 \mathrm{GeV}$. We set the factorization scale equal to the renormalization scale and denote this common scale by $\mu$. Again we use the MRST2002 approximate NNLO parton distribution functions [34] (results using the CTEQ6M distributions [41] are similar [14]). We plot a large range in scale and see that the higher-order soft-gluon corrections greatly decrease the scale dependence of the cross section. The NNLO-NNNLL and the NNNLONNLL curves are relatively flat. The NNNLO-NNLL result displays very little variation and it approaches the scale independence expected of a physical cross section.

\section{Conclusions}

In this paper, I presented a unified approach to calculating the NNNLO soft-gluon corrections for hard-scattering processes in hadron-hadron and lepton-hadron collisions in the $\overline{\mathrm{MS}}$ scheme in either 1PI or PIM kinematics. The master formulas given in the paper allow explicit calculations for any process, with either simple or complex color flows, keeping in general the factorization and renormalization scales separate and the color factors explicit. Detailed results, illustrating the use of the master formulas, were given to NLL accuracy for charged Higgs production via bottom-gluon fusion at the LHC, and to NNLL accuracy for top quark production via quark-antiquark annihilation at the Tevatron.

The NNLO and NNNLO corrections increase theoretical accuracy and diminish the dependence on the factorization and renormalization scales, and thus are essential in further calculations of QCD corrections for many hard-scattering processes, both in their own right and as backgrounds which may be particularly important in searching for the Higgs boson and supersymmetric particles, as well as other processes that signal new physics beyond the Standard Model.

\section{Appendix}

The $\beta$ function is given by

$$
\beta\left(\alpha_{s}\right) \equiv \mu d \ln g / d \mu=-\beta_{0} \alpha_{s} /(4 \pi)-\beta_{1} \alpha_{s}^{2} /(4 \pi)^{2}-\beta_{2} \alpha_{s}^{3} /(4 \pi)^{3}+\cdots,
$$

where $g^{2}=4 \pi \alpha_{s}$, with $\beta_{0}=\left(11 C_{A}-2 n_{f}\right) / 3, \beta_{1}=34 C_{A}^{2} / 3-2 n_{f}\left(C_{F}+5 C_{A} / 3\right)$, and

$$
\beta_{2}=\frac{2857}{54} C_{A}^{3}+\left(C_{F}^{2}-\frac{615}{54} C_{F} C_{A}-\frac{1415}{54} C_{A}^{2}\right) n_{f}+\left(\frac{33}{27} C_{F}+\frac{79}{54} C_{A}\right) n_{f}^{2} .
$$

Note that

$$
\begin{gathered}
\alpha_{s}(\mu)=\alpha_{s}\left(\mu_{R}\right)\left[1-\frac{\beta_{0}}{4 \pi} \alpha_{s}\left(\mu_{R}\right) \ln \left(\frac{\mu^{2}}{\mu_{R}^{2}}\right)+\frac{\beta_{0}^{2}}{16 \pi^{2}} \alpha_{s}^{2}\left(\mu_{R}\right) \ln ^{2}\left(\frac{\mu^{2}}{\mu_{R}^{2}}\right)-\frac{\beta_{1}}{16 \pi^{2}} \alpha_{s}^{2}\left(\mu_{R}\right) \ln \left(\frac{\mu^{2}}{\mu_{R}^{2}}\right)\right. \\
+\cdots] .
\end{gathered}
$$

The various quantities used in the NNNLO expression, Eq. (5.1), are given by the following expressions. For the quantities $X_{3}, X_{2}, X_{1}, X_{0}$, we have

$$
X_{3}=\frac{\beta_{0}}{12} c_{3}-\sum_{j} C_{j} \frac{\beta_{0}}{24},
$$




$$
\begin{gathered}
X_{2}=-\frac{\beta_{0}}{4} T_{2}+\frac{\beta_{0}}{8} c_{3} \ln \left(\frac{\mu_{R}^{2}}{M^{2}}\right)+c_{3} \frac{K}{4}-\sum_{j} \frac{\beta_{0}}{8} B_{j}^{(1)}, \\
X_{1}=c_{2} c_{1}-\zeta_{2} c_{3} c_{2}+\zeta_{3} c_{3}^{2}+\frac{\beta_{0}}{4} \zeta_{2} c_{3}-\sum_{j} C_{j} \frac{\beta_{0}}{8} \zeta_{2}-C_{D_{0}}^{(2)},
\end{gathered}
$$

and

$$
\begin{aligned}
X_{0}= & \frac{\beta_{0}}{4} c_{1} \ln \left(\frac{\mu_{R}^{2}}{M^{2}}\right)+\frac{d_{\alpha_{s}}}{16}\left[-\frac{\beta_{0}^{2}}{2} \ln ^{2}\left(\frac{\mu_{R}^{2}}{M^{2}}\right)-\beta_{0}^{2} \ln \left(\frac{\mu_{R}^{2}}{M^{2}}\right) \ln \left(\frac{M^{2}}{s}\right)+\beta_{1} \ln \left(\frac{\mu_{R}^{2}}{M^{2}}\right)\right] \\
& +\frac{\beta_{0}}{6} \zeta_{3} c_{3}-\frac{\beta_{0}}{4} \zeta_{2} T_{2}+\frac{\beta_{0}}{8} \zeta_{2} c_{3} \ln \left(\frac{\mu_{R}^{2}}{M^{2}}\right)+\sum_{i} C_{i} \frac{\zeta_{2}}{2} K-\sum_{i} \gamma_{i / i}^{\prime(2)} \ln \left(\frac{\mu_{F}^{2}}{M^{2}}\right) \\
& +\sum_{i} \frac{\beta_{0}}{8}\left[\gamma_{i}^{(1)}-C_{i} \ln \left(\frac{-t_{i}}{M^{2}}\right)\right]\left[\ln ^{2}\left(\frac{\mu_{F}^{2}}{M^{2}}\right)+2 \ln \left(\frac{\mu_{F}^{2}}{M^{2}}\right) \ln \left(\frac{M^{2}}{s}\right)\right] \\
& +\sum_{i} C_{i} \frac{K}{2} \ln \left(\frac{-t_{i}}{M^{2}}\right) \ln \left(\frac{\mu_{F}^{2}}{M^{2}}\right)-\sum_{j} C_{j} \frac{\beta_{0}}{12} \zeta_{3}-\sum_{j} C_{j} \zeta_{2} \frac{K}{4}-\sum_{j} \frac{\beta_{0}}{8} \zeta_{2} B_{j}^{(1)}+R^{\prime(2)} .(A .)
\end{aligned}
$$

The quantity $R^{\prime(2)}$ at the end of the above equation stands for the virtual two-loop corrections $R^{(2)}$ in Eq. (4.1) minus $\zeta$ terms and scale terms. To be precise

$$
\begin{aligned}
R^{(2)}= & R^{\prime(2)}+\frac{1}{2} c_{1}^{2}-\frac{\zeta_{2}}{2} c_{2}^{2}+\frac{1}{4} \zeta_{2}^{2} c_{3}^{2}+\zeta_{3} c_{3} c_{2}-\frac{3}{4} \zeta_{4} c_{3}^{2}+\frac{\beta_{0}}{4} c_{1} \ln \left(\frac{\mu_{R}^{2}}{M^{2}}\right) \\
& +\frac{d_{\alpha_{s}}}{16}\left[-\frac{\beta_{0}^{2}}{2} \ln ^{2}\left(\frac{\mu_{R}^{2}}{M^{2}}\right)-\beta_{0}^{2} \ln \left(\frac{\mu_{R}^{2}}{M^{2}}\right) \ln \left(\frac{M^{2}}{s}\right)+\beta_{1} \ln \left(\frac{\mu_{R}^{2}}{M^{2}}\right)\right] \\
& -\sum_{i} \gamma_{i / i}^{\prime(2)} \ln \left(\frac{\mu_{F}^{2}}{M^{2}}\right)+\sum_{i} C_{f_{i}} \frac{K}{2} \ln \left(\frac{-t_{i}}{M^{2}}\right) \ln \left(\frac{\mu_{F}^{2}}{M^{2}}\right) \\
& +\sum_{i} \frac{\beta_{0}}{8}\left[\gamma_{i}^{(1)}-C_{f_{i}} \ln \left(\frac{-t_{i}}{M^{2}}\right)\right]\left[\ln ^{2}\left(\frac{\mu_{F}^{2}}{M^{2}}\right)+2 \ln \left(\frac{\mu_{F}^{2}}{M^{2}}\right) \ln \left(\frac{M^{2}}{s}\right)\right] .
\end{aligned}
$$

Also

$$
\begin{gathered}
Y_{i}^{(1)}=2 A_{i}^{(3)}+C_{i} \beta_{0}\left[\frac{\beta_{0}}{8} \ln ^{2}\left(\frac{\mu_{F}^{2}}{\mu_{R}^{2}}\right)-\frac{K}{2} \ln \left(\frac{\mu_{F}^{2}}{\mu_{R}^{2}}\right)\right] \\
+C_{i} \frac{\beta_{1}}{8}\left[\ln \left(\frac{\mu_{R}^{2}}{M^{2}}\right)+\ln \left(\frac{M^{2}}{s}\right)+1+2 \ln \left(\frac{-t_{i}}{M^{2}}\right)\right], \\
Y_{j}^{(1)=}-\frac{\beta_{0}}{2} B_{j}^{(2)}-A_{j}^{(3)}-C_{j} \frac{\beta_{0}^{2}}{16} \ln ^{2}\left(\frac{\mu_{R}^{2}}{M^{2}}\right)+\frac{\beta_{0}}{8}\left[-\beta_{0} B_{j}^{(1)}-2 C_{j} K\right] \ln \left(\frac{\mu_{R}^{2}}{M^{2}}\right) \\
+\frac{C_{j}}{8} \zeta_{2} \beta_{0}^{2}+\frac{\beta_{1}}{16}\left[-C_{j} \ln \left(\frac{\mu_{R}^{2}}{M^{2}}\right)+2 C_{j}+B_{j}^{(1)}+2 C_{j} \ln \left(\frac{M^{2}}{s}\right)\right],
\end{gathered}
$$




$$
\begin{aligned}
Y_{i}^{(0)}= & -C_{i} \frac{\beta_{0}^{2}}{48}\left[\ln ^{3}\left(\frac{\mu_{F}^{2}}{\mu_{R}^{2}}\right)+\ln ^{3}\left(\frac{\mu_{R}^{2}}{M^{2}}\right)\right]+\frac{C_{i}}{32}\left(\beta_{1}+4 \beta_{0} K\right)\left[\ln ^{2}\left(\frac{\mu_{F}^{2}}{\mu_{R}^{2}}\right)-\ln ^{2}\left(\frac{\mu_{R}^{2}}{M^{2}}\right)\right] \\
& -C_{i} \frac{\beta_{0}}{4} K\left[2 \ln \left(\frac{-t_{i}}{M^{2}}\right)+\ln \left(\frac{M^{2}}{s}\right)\right] \ln \left(\frac{\mu_{R}^{2}}{M^{2}}\right)-C_{i} \frac{\beta_{1}}{16}\left[1+2 \ln \left(\frac{-t_{i}}{M^{2}}\right)\right] \ln \left(\frac{\mu_{R}^{2}}{M^{2}}\right) \\
& -\frac{\beta_{0}^{2}}{16}\left[4 \gamma_{i}^{(1)}+2 C_{i}-\beta_{0} d_{\alpha_{s}}+C_{i} \frac{\beta_{1}}{\beta_{0}^{2}}+C_{i} \ln \left(\frac{M^{2}}{s}\right)\right] \ln \left(\frac{M^{2}}{s}\right) \ln \left(\frac{\mu_{R}^{2}}{M^{2}}\right) \\
& -\nu_{i}^{(2)} \frac{\beta_{0}}{2} \ln \left(\frac{\mu_{R}^{2}}{M^{2}}\right)-A_{i}^{(3)} \ln \left(\frac{\mu_{F}^{2}}{M^{2}}\right)-\nu_{i}^{(3)}-\frac{C_{i}}{6} \beta_{0}^{2} \ln ^{3}\left(\frac{-t_{i}}{M^{2}}\right)-\frac{C_{i}}{4} \beta_{0}\left(\beta_{0}+2 K\right) \ln ^{2}\left(\frac{-t_{i}}{M^{2}}\right) \\
& -\left(\beta_{0} \nu_{i}^{(2)}+2 A_{i}^{(3)}\right) \ln \left(\frac{-t_{i}}{M^{2}}\right)-\frac{\beta_{0}^{2}}{48} C_{i} \ln ^{3}\left(\frac{M^{2}}{s}\right)-\frac{C_{i}}{32}\left(\beta_{1}+4 \beta_{0} K\right) \ln ^{2}\left(\frac{M^{2}}{s}\right) \\
& +\frac{\beta_{0}^{2}}{4}\left[\frac{C_{i}}{2} \ln \left(\frac{-t_{i}}{M^{2}}\right)-\gamma_{i}^{(1)}-\frac{C_{i}}{4}+\frac{\beta_{0}}{4} d_{\alpha_{s}}\right] \ln ^{2}\left(\frac{M^{2}}{s}\right) \\
& +\left[-A_{i}^{(3)}-\nu_{i}^{(2)} \frac{\beta_{0}}{2}-C_{i} K \frac{\beta_{0}}{2} \ln \left(\frac{-t_{i}}{M^{2}}\right)+\frac{\beta_{1} \beta_{0}}{32} d_{\alpha_{s}}-C_{i} \frac{\beta_{1}}{16}-\frac{\beta_{1}}{8} \gamma_{i}^{(1)}\right] \ln \left(\frac{M^{2}}{s}\right), \quad(\mathrm{A} .11)
\end{aligned}
$$

and

$$
\begin{aligned}
Y_{j}^{(0)}= & \frac{\beta_{0}}{4}\left[-2 B_{j}^{(2)}-2 \nu_{j}^{(2)}-\left(\frac{\beta_{0}}{2}\left(2 B_{j}^{(1)}+C_{j}\right)+C_{j} K\right) \ln \left(\frac{M^{2}}{s}\right)-C_{j} \frac{\beta_{0}}{4} \ln ^{2}\left(\frac{M^{2}}{s}\right)\right] \ln \left(\frac{\mu_{R}^{2}}{M^{2}}\right) \\
& -\frac{\beta_{1}}{16}\left[B_{j}^{(1)}+C_{j}+C_{j} \ln \left(\frac{M^{2}}{s}\right)\right] \ln \left(\frac{\mu_{R}^{2}}{M^{2}}\right)-B_{j}^{(3)}-\nu_{j}^{(3)}-\frac{7}{48} C_{j} \beta_{0}^{2} \ln ^{3}\left(\frac{M^{2}}{s}\right) \\
& +\frac{\beta_{0}}{16}\left(-5 C_{j} \beta_{0}-4 \beta_{0} B_{j}^{(1)}+2 C_{j} K\right) \ln ^{2}\left(\frac{M^{2}}{s}\right)-\frac{\beta_{1}}{32} C_{j} \ln ^{2}\left(\frac{M^{2}}{s}\right) \\
& +\left[\frac{\beta_{0}}{2} \nu_{j}^{(2)}-A_{j}^{(3)}-\frac{\beta_{1}}{16}\left(2 B_{j}^{(1)}+C_{j}\right)\right] \ln \left(\frac{M^{2}}{s}\right) .
\end{aligned}
$$

Also we have defined,

$$
\begin{gathered}
M^{c}=\operatorname{tr}\left[H^{(1)} \Gamma_{S}^{(1) \dagger} S^{(0)}+H^{(1)} S^{(0)} \Gamma_{S}^{(1)}+H^{(0)} \Gamma_{S}^{(1) \dagger} S^{(1)}+H^{(0)} S^{(1)} \Gamma_{S}^{(1)}\right] \\
K_{1}^{c}=\operatorname{tr}\left[H^{(2)} \Gamma_{S}^{(1) \dagger} S^{(0)}+H^{(0)} S^{(2)} \Gamma_{S}^{(1)}+H^{(2)} S^{(0)} \Gamma_{S}^{(1)}\right. \\
+H^{(0)} \Gamma_{S}^{(1) \dagger} S^{(2)}+H^{(1)} \Gamma_{S}^{(1) \dagger} S^{(1)}+H^{(1)} S^{(1)} \Gamma_{S}^{(1)} \\
+H^{(1)} \Gamma_{S}^{(2) \dagger} S^{(0)}+H^{(1)} S^{(0)} \Gamma_{S}^{(2)}+H^{(0)} \Gamma_{S}^{(2) \dagger} S^{(1)} \\
\left.+H^{(0)} S^{(1)} \Gamma_{S}^{(2)}+H^{(0)} \Gamma_{S}^{(3) \dagger} S^{(0)}+H^{(0)} S^{(0)} \Gamma_{S}^{(3)}\right] \\
K_{2}^{c}=\operatorname{tr}\left[H^{(1)}\left(\Gamma_{S}^{(1) \dagger}\right)^{2} S^{(0)}+H^{(0)}\left(\Gamma_{S}^{(1) \dagger}\right)^{2} S^{(1)}+\left(H^{(1)} S^{(0)}+H^{(0)} S^{(1)}\right)\left(\Gamma_{S}^{(1)}\right)^{2}\right. \\
+2 H^{(1)} \Gamma_{S}^{(1) \dagger} S^{(0)} \Gamma_{S}^{(1)}+2 H^{(0)} \Gamma_{S}^{(1) \dagger} S^{(1)} \Gamma_{S}^{(1)}+2 H^{(0)} \Gamma_{S}^{(1) \dagger} \Gamma_{S}^{(2) \dagger} S^{(0)} \\
\left.+2 H^{(0)} S^{(0)} \Gamma_{S}^{(1)} \Gamma_{S}^{(2)}+2 H^{(0)} \Gamma_{S}^{(2) \dagger} S^{(0)} \Gamma_{S}^{(1)}+2 H^{(0)} \Gamma_{S}^{(1) \dagger} S^{(0)} \Gamma_{S}^{(2)}\right]
\end{gathered}
$$


and

$$
\begin{aligned}
K_{3}^{c}= & \operatorname{tr}\left[H^{(0)}\left(\Gamma_{S}^{(1) \dagger}\right)^{3} S^{(0)}+H^{(0)} S^{(0)}\left(\Gamma_{S}^{(1)}\right)^{3}\right. \\
& \left.+3 H^{(0)}\left(\Gamma_{S}^{(1) \dagger}\right)^{2} S^{(0)} \Gamma_{S}^{(1)}+3 H^{(0)} \Gamma_{S}^{(1) \dagger} S^{(0)}\left(\Gamma_{S}^{(1)}\right)^{2}\right] .
\end{aligned}
$$

\section{References}

[1] The QCD/SM Working Group: Summary Report, in Les Houches 2003, Physics at TeV colliders, p. 291, hep-ph/0403100, and references therein.

[2] G. Sterman, Nucl. Phys. B 281, 310 (1987).

[3] S. Catani and L. Trentadue, Nucl. Phys. B 327, 323 (1989).

[4] H. Contopanagos, E. Laenen, and G. Sterman, Nucl. Phys. B 484, 303 (1997) hep-ph/9604313.

[5] N. Kidonakis and G. Sterman, Phys. Lett. B 387, 867 (1996); Nucl. Phys. B 505, 321 (1997) hep-ph/9705234.

[6] N. Kidonakis, G. Oderda, and G. Sterman, Nucl. Phys. B 525, 299 (1998) hep-ph/9801268; Nucl. Phys. B 531, 365 (1998) hep-ph/9803241.

[7] E. Laenen, G. Oderda, and G. Sterman, Phys. Lett. B 438, 173 (1998) hep-ph/9806467.

[8] N. Kidonakis, in DPF 2004, Int. J. Mod. Phys. A 20, 3726 (2005) hep-ph/0410116; in DIS 2005, hep-ph/0506299.

[9] N. Kidonakis, Phys. Rev. D 64, 014009 (2001) hep-ph/0010002.

[10] N. Kidonakis, Mod. Phys. Lett. A 19, 405 (2004) hep-ph/0401147, and references therein.

[11] N. Kidonakis and A. Sabio Vera, JHEP 02, 027 (2004) hep-ph/0311266; R.J. Gonsalves, N. Kidonakis, and A. Sabio Vera, hep-ph/0507317; N. Kidonakis and V. Del Duca, Phys. Lett. B 480, 87 (2000) hep-ph/9911460.

[12] N. Kidonakis and J.F. Owens, Phys. Rev. D 61, 094004 (2000) hep-ph/9912388; Int. J. Mod. Phys. A 19, 149 (2004) hep-ph/0307352.

[13] N. Kidonakis, E. Laenen, S. Moch, and R. Vogt, Phys. Rev. D 64, 114001 (2001) hep-ph/0105041; N. Kidonakis, in DPF 2000, Int. J. Mod. Phys. A 16, s1A, 363 (2001) hep-ph/0009013.

[14] N. Kidonakis and R. Vogt, Phys. Rev. D 68, 114014 (2003) hep-ph/0308222; Eur. Phys. J. C 33, s466 (2004) hep-ph/0309045; in DPF 2004, Int. J. Mod. Phys. A 20, 3171 (2005) hep-ph/0410367. 
[15] N. Kidonakis, E. Laenen, S. Moch, and R. Vogt, Phys. Rev. D 67, 074037 (2003) hep-ph/0212173; Nucl. Phys. A 715, 549 (2003) hep-ph/0208119; N. Kidonakis and R. Vogt, Eur. Phys. J. C 36, 201 (2004) hep-ph/0401056.

[16] E. Laenen and S. Moch, Phys. Rev. D 59, 034027 (1999) hep-ph/9809550.

[17] N.Y. Ivanov, Nucl. Phys. B 615, 266 (2001) hep-ph/0104301.

[18] N. Kidonakis, JHEP 05, 011 (2005) hep-ph/0412422; in DIS 2005, hep-ph/0505271.

[19] N. Kidonakis and J.F. Owens, Phys. Rev. D 63, 054019 (2001) hep-ph/0007268.

[20] Beyond the Standard Model Working Group: Summary Report, in Les Houches 2003, Physics at TeV colliders, p. 171, hep-ph/0402295, and references therein.

[21] The Higgs Working Group: Summary Report, in Les Houches 2003, Physics at TeV colliders, p. 1, hep-ph/0406152, and references therein.

[22] A. Belyaev and N. Kidonakis, Phys. Rev. D 65, 037501 (2002) hep-ph/0102072; N. Kidonakis and A. Belyaev, JHEP 12, 004 (2003) hep-ph/0310299.

[23] N. Kidonakis, Int. J. Mod. Phys. A 19, 1793 (2004) hep-ph/0303186; in DIS 2004, hep-ph/0306125, hep-ph/0307207.

[24] N. Kidonakis, Int. J. Mod. Phys. A 15, 1245 (2000) hep-ph/9902484, and references therein.

[25] C.F. Berger, Phys. Rev. D 66, 116002 (2002) hep-ph/0209107.

[26] S. Moch, J.A.M. Vermaseren, and A. Vogt, hep-ph/0506288.

[27] J. Kodaira and L. Trentadue, Phys. Lett. B 112, 66 (1982).

[28] J. Botts and G. Sterman, Nucl. Phys. B 325, 62 (1989).

[29] N. Kidonakis, hep-ph/0208056 in DIS 2003, hep-ph/0307145.

[30] A. Belyaev, D. Garcia, J. Guasch, and J. Sola, Phys. Rev. D 65, 031701 (2002) hep-ph/0105053; JHEP 06, 059 (2002) hep-ph/0203031.

[31] S. Zhu, Phys. Rev. D 67, 075006 (2003) hep-ph/0112109.

[32] T. Plehn, Phys. Rev. D 67, 014018 (2003) hep-ph/0206121.

[33] E.L. Berger, T. Han, J. Jiang, and T. Plehn, Phys. Rev. D 71, 115012 (2005) hep-ph/0312286.

[34] A.D. Martin, R.G. Roberts, W.J. Stirling, and R.S. Thorne, Eur. Phys. J. C 28, 455 (2003) hep-ph/0211080.

[35] D. de Florian and W. Vogelsang, Phys. Rev. D 71, 114004 (2005) hep-ph/0501258. 
[36] CDF Coll., hep-ex/0504053 Phys. Rev. D 72, 032002 (2005) hep-ex/0506001.

[37] D0 Coll., hep-ex/0504043; hep-ex/0504058; hep-ex/0505082,

[38] W. Wagner, Rept. Prog. Phys. 68, 2409 (2005) hep-ph/0507207, and references therein.

[39] N. Kidonakis and J. Smith, Phys. Rev. D 51, 6092 (1995) hep-ph/9502341.

[40] W. Beenakker, W.L. van Neerven, R. Meng, G.A. Schuler, and J. Smith, Nucl. Phys. B 351, 507 (1991).

[41] J. Pumplin, D.R. Stump, J. Huston, H.L. Lai, P. Nadolsky, and W.K. Tung, JHEP 07, 012 (2002) hep-ph/0201195. 